\title{
Semiexclusive dilepton production in proton-proton collisions with one forward proton measurement at the LHC
}

\author{
Antoni Szczurek $\oplus^{1, *}$ Barbara Linek $\oplus^{2, \dagger}$ and Marta Łuszczak $\odot^{2, \ddagger}$ \\ ${ }^{1}$ Institute of Nuclear Physics, Polish Academy of Sciences, \\ ul. Radzikowskiego 152, PL-31-342 Kraków, Poland \\ ${ }^{2}$ College of Natural Sciences, Institute of Physics, University of Rzeszów, \\ ul. Pigonia 1, PL-35-959 Rzeszów, Poland
}

(Received 26 July 2021; accepted 20 September 2021; published 12 October 2021)

\begin{abstract}
We discuss photon-photon fusion mechanisms of dilepton production in proton-proton collisions with rapidity gap in the main detector and one forward proton in the forward proton detectors. This is relevant for the LHC measurements by ATLAS + AFP and CMS + PPS. Transverse momenta of the intermediate photons are taken into account and photon fluxes are expressed in terms of proton electromagnetic form factors and structure functions. Differential distributions in $\xi_{1 / 2}, M_{l l}, Y_{l l}, p_{t, l l}, M_{R}$ are shown, and the competition of different mechanisms is discussed. Both double-elastic and singledissociative processes are included in the analysis. Different parametrizations of the structure functions are used. We discuss also mechanism with one forward $\Delta^{+}$isobar or other proton resonances in the final state. The role of several cuts is studied. We also use the sUPERCHIC generator and compare corresponding results to the results of our codes. The soft rapidity gap survival factor is calculated for each contribution separately. The gap survival factor for the single-dissociative mechanism due to minijet emission into the main detector is calculated in addition. It depends on the type of contribution (fully elastic, single dissociation, double dissociation). The soft rapidity gap survival factor for the case of single proton measurement is significantly smaller than that for the inclusive case (no proton measurement). We find only weak dependence on the invariant mass of the dilepton system as well as the lepton pair transverse momentum and sizeable dependence on the pair rapidity. The latter effect is rather difficult to identify experimentally.
\end{abstract}

DOI: 10.1103/PhysRevD.104.074009

\section{INTRODUCTION}

The production of dilepton pairs via photon-photon fusion was studied both experimentally $[1,2]$ and theoretically $[3,4]$.

In [4] we proposed how to include transverse momenta of fusing virtual photons. The formalism was used to calculate distributions of several observables related to leptons.

Till recently forward going protons were not measured. However, recently both CMS + TOTEM [2] and ATLAS [1] measured the cases with one proton in PPS (Precision

\footnotetext{
*antoni.szczurek@ifj.edu.pl

Also at University of Rzeszów, PL-35-959 Rzeszów, Poland.

†basialinek@gmail.com

†uszczak@ur.edu.pl

Published by the American Physical Society under the terms of the Creative Commons Attribution 4.0 International license. Further distribution of this work must maintain attribution to the author(s) and the published article's title, journal citation, and DOI. Funded by SCOAP ${ }^{3}$.
}

Proton Spectrometer) or AFP (ATLAS Forward Proton), respectively. This automatically selects a fully exclusive process or processes with single proton dissociation. The CMS collaboration measured only a few events as a $p_{t}>50 \mathrm{GeV}$ cut was imposed there, whereas the ATLAS collaboration had a $p_{t}>15 \mathrm{GeV}$ cut. So the ATLAS collaboration could obtain even some distributions.

Only recently the CMS collaboration [2] and very recently the ATLAS collaboration [1] presented results with at least one proton measured in forward direction. The experimental apparatus allows us to measure only very forward protons. In theoretical calculations one has to impose experimental limits on so-called $\xi$ variables (longitudinal momentum fraction loss) $[1,2]$. The limited acceptance of the forward detectors causes that the cross section is considerably reduced compared to the case when only leptons are measured, as will be discussed here.

Here, we use the formalism developed in [4,5], which allows us to calculate the cross section differential also in $M_{X}$ or $M_{Y}$, masses of the excited proton remnants. In [6,7] it was discussed how to calculate gap survival factor which is related to emission of (mini)jets produced in a 


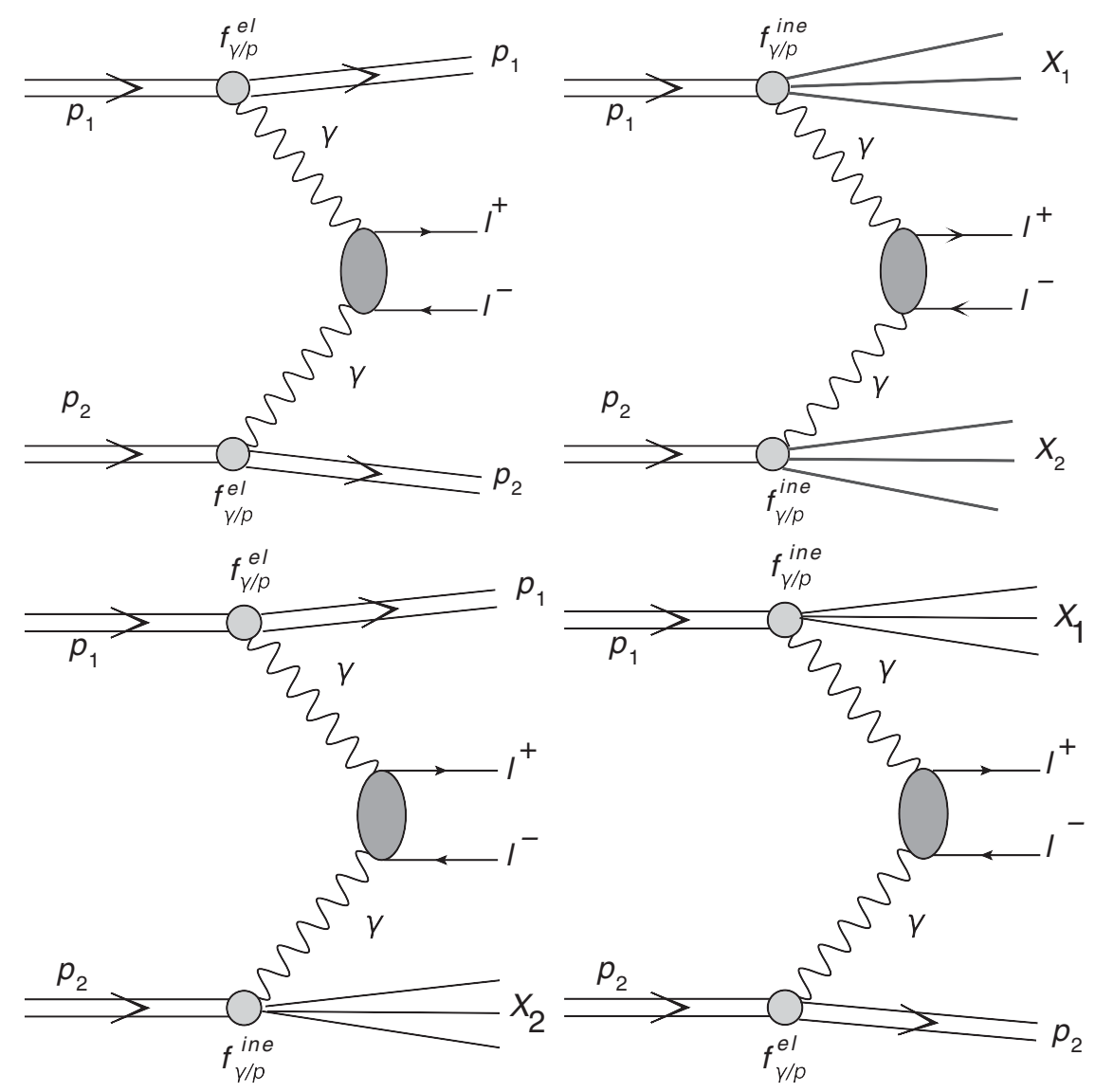

FIG. 1. Four different categories of $\gamma \gamma$ fusion mechanisms of dilepton production in proton-proton collisions.

DIS (deep-inelastic scattering) process associted with $W^{+} W^{-}$and $t \bar{t}$ production, respectively. We shall repeat such a calculation also here for $\mu^{+} \mu^{-}$production. The absorption for double-elastic contribution was studied, e.g., in $[8,9]$ using the momentum space formalism. The impact parameter approach can be found, e.g., in [10].

The same processes and a similar formalism were implemented in the recent version of the SUPERCHIC 4 generator [11]. This code generates events with four momenta of outgoing particles (leptons, protons, jets). The authors of SUPERCHIC implemented also soft absorption effects in the form of kinematics-dependent gap survival factor. In the present study we shall use also the SUPERCHIC 4 code for comparison and in order to estimate the soft rapidity gap survival probability.

It is the aim of this paper to investigate what is the influence of the measurement of one proton (limited longitudinal fraction of the outgoing proton) on different leptonic differential distributions, not only those measured by the ATLAS collaboration. We wish to investigate also how the gap survival factor (soft and the one due to minijet production) changes when proton is measured compared to the inclusive case (no proton measurement). We shall try to include all processes in the state-of-art approach taking into account transverse momenta of intermediate photons.

\section{SKETCH OF THE FORMALISM}

In general, there are four categories of the $\gamma \gamma$ processes as shown in Fig. 1. We shall call them elastic-elastic, inelasticinelastic, elastic-inelastic, and inelastic-elastic processes. The first one will be also called double elastic and the second one double inelastic for brevity.

In the $k_{T}$-factorization approach $[3,4]$, the cross section for production of $l^{+} l^{-}$can be written in the form

$$
\begin{aligned}
\frac{d \sigma^{(i, j)}}{d y_{1} d y_{2} d^{2} \boldsymbol{p}_{1} d^{2} \boldsymbol{p}_{2}}= & \int \frac{d^{2} \boldsymbol{q}_{1}}{\pi \boldsymbol{q}_{1}^{2}} \frac{d^{2} \boldsymbol{q}_{2}}{\pi \boldsymbol{q}_{2}^{2}} \mathcal{F}_{\gamma^{*} / A}^{(i)}\left(x_{1}, \boldsymbol{q}_{1}\right) \\
& \times \mathcal{F}_{\gamma^{*} / B}^{(j)}\left(x_{2}, \boldsymbol{q}_{2}\right) \frac{d \sigma^{*}\left(p_{1}, p_{2} ; \boldsymbol{q}_{1}, \boldsymbol{q}_{2}\right)}{d y_{1} d y_{2} d^{2} \boldsymbol{p}_{1} d^{2} \boldsymbol{p}_{2}},
\end{aligned}
$$

where the indices $i, j \in\{\mathrm{el}, \mathrm{in}\}$ denote elastic or inelastic final states. Here the photon flux for inelastic case is integrated over the mass of the remnant. 
The longitudinal momentum fractions of photons are obtained from the rapidities and transverse momenta of final state $l^{+} l^{-}$as

$$
\begin{aligned}
& x_{1}=\sqrt{\frac{\boldsymbol{p}_{1}^{2}+m_{l}^{2}}{s}} e^{+y_{1}}+\sqrt{\frac{\boldsymbol{p}_{2}^{2}+m_{l}^{2}}{s}} e^{+y_{2}}, \\
& x_{2}=\sqrt{\frac{\boldsymbol{p}_{1}^{2}+m_{l}^{2}}{s}} e^{-y_{1}}+\sqrt{\frac{\boldsymbol{p}_{2}^{2}+m_{l}^{2}}{s}} e^{-y_{2}} .
\end{aligned}
$$

The integrated fluxes for elastic and inelastic processes can be found in $[4,5]$.

Then the four-momenta of intermediate photons can be written as

$$
\begin{aligned}
& q_{1} \approx\left(x_{1} \frac{\sqrt{s}}{2}, \vec{q}_{1 t}, x_{1} \frac{\sqrt{s}}{2}\right), \\
& q_{2} \approx\left(x_{2} \frac{\sqrt{s}}{2}, \vec{q}_{2 t},-x_{2} \frac{\sqrt{s}}{2}\right) .
\end{aligned}
$$

If one is interested in modeling what happens with the proton remnant then the formalism must be somewhat extended. Then the unintegrated inelastic photon distribution (flux) can be written as

$$
\mathcal{F}_{\text {ine }}\left(x, q_{t}^{2}\right)=\int d M^{2} \frac{d \mathcal{F}_{\text {ine }}}{d M^{2}}\left(x, q_{t}^{2}, M^{2}\right),
$$

where $\frac{d \mathcal{F}_{\text {ine }}}{d M^{2}}\left(x, q_{t}^{2}, M^{2}\right)$ is a more differential photon distribution in the proton. In the following we shall call it doubly unintegrated photon distribution (flux). The latter distribution was used to calculate differential distributions for production of $W^{+} W^{-}$[6] or $t \bar{t}$ [7] pairs with rapidity gap at midrapidities.

In principle, proton can be emitted also from the remnant system. This requires modeling of remnant fragmentation which is not fully under control. Such protons carry typically much reduced longitudinal momentum fraction $x_{i}$ such that $\xi_{i}=1-x_{i}>0.1$; i.e., it cannot be measured in the Roman pots of the ATLAS or CMS experiments.

Only the diffractive mechanism shown in Fig. 2 could lead to $\xi_{i}<0.1$. However, the diffractive mechanism happens only in about $10 \%$ of all cases as was measured at HERA [12]. In addition, the pomeron remnant would destroy the rapidity gap. Such a process was not discussed in the context of $l^{+} l^{-}$production in $p p$ collisions with a rapidity gap requirement. Also the diffractive photon distribution in pomeron was not discussed. One may expect the following:

$$
\frac{d \mathcal{F}_{\text {diff }}}{d M^{2}}\left(x, q_{t}^{2}, M^{2}\right) \ll \frac{d \mathcal{F}_{\text {ine }}}{d M^{2}}\left(x, q_{t}^{2}, M^{2}\right) .
$$

In addition, the rapidity gap veto condition would almost totally eliminate contribution of such processes in the context of forward proton measurement discussed in the present paper.

The discussion above shows that the double-dissociative contribution would be to large extent eliminated.

The ATLAS collaboration analysis imposes the consistency requirements:

$$
\xi_{1}=\xi_{l l}^{+}, \quad \xi_{2}=\xi_{l l}^{-} .
$$

The longitudinal momentum fractions of the photons were calculated in the ATLAS analysis as follows:

$$
\begin{aligned}
& \xi_{l l}^{+}=\left(M_{l l} / \sqrt{s}\right) \exp \left(+Y_{l l}\right), \\
& \xi_{l l}^{-}=\left(M_{l l} / \sqrt{s}\right) \exp \left(-Y_{l l}\right) .
\end{aligned}
$$

Only lepton variables enter the formula. This is only approximate formula which can be improved, if necessary. We will use the same formula in our analysis.

The inelastic photon fluxes are expressed in terms of so-called proton structure functions [4]. The arguments of the structure functions, called here Bjorken- $x$ for brevity, can be calculated as

$$
x_{B j, 1 / 2}=\frac{Q_{1 / 2}^{2}}{Q_{1 / 2}^{2}+M_{X / Y}^{2}-m_{p}^{2}},
$$

where $Q_{1 / 2}^{2}$ are photon virtualities and $M_{X / Y}$ are masses of proton remnants.

\section{RESULTS}

In the calculations described below we shall take typical cuts on dileptons: $-2.5<y_{1}, y_{2}<2.5$ and $p_{1 t}, p_{2 t}>15 \mathrm{GeV}$.
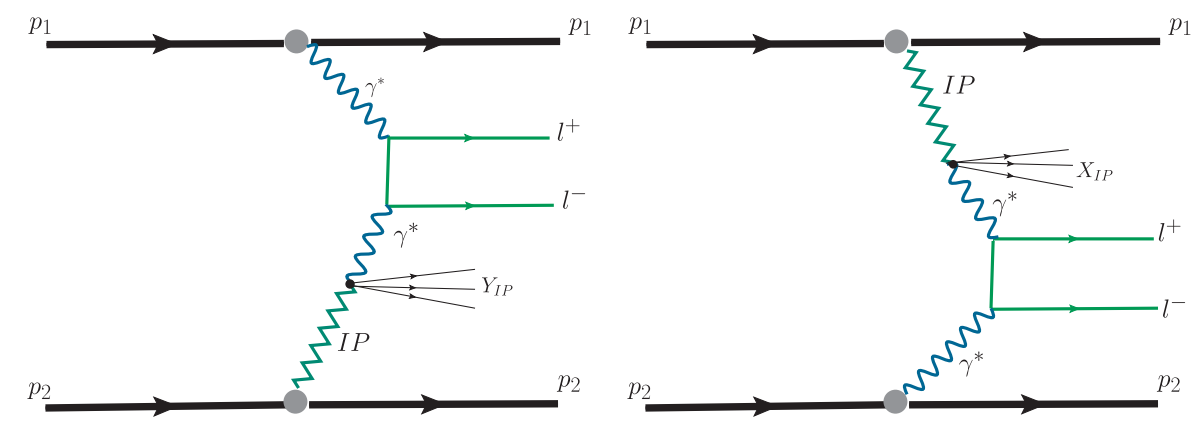

FIG. 2. Diffractive mechanisms of dilepton production in proton-proton collisions. 


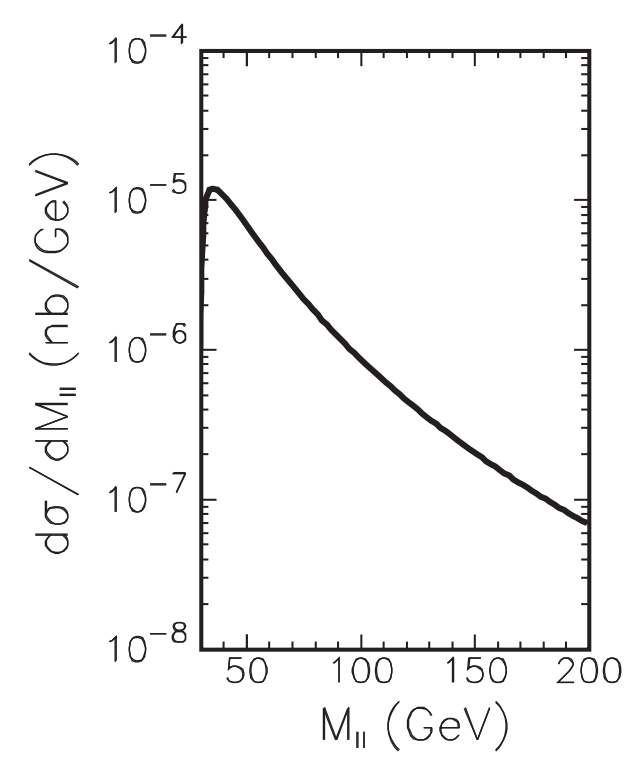

FIG. 3. Dimuon invariant mass distribution of the doubleelastic mechanism.

We shall show also results with extra cuts on $\xi_{l l}^{+}$or $\xi_{l l}^{-}$. In the following we shall not exclude mass window around $Z$-boson mass $m_{Z}$ and/or lepton acoplanarity, as was done in [1].

\section{A. Double-elastic contribution}

We start from presenting results for the double-elastic contribution. Here the flux of photons can be expressed in terms of elastic form factors of proton. We take typical cuts for such a measurement [1]: $p_{t}>15 \mathrm{GeV}$ and $-2.5<y_{1}, y_{2}<2.5$. In the following we limit ourself to dimuon production. The results for electron production

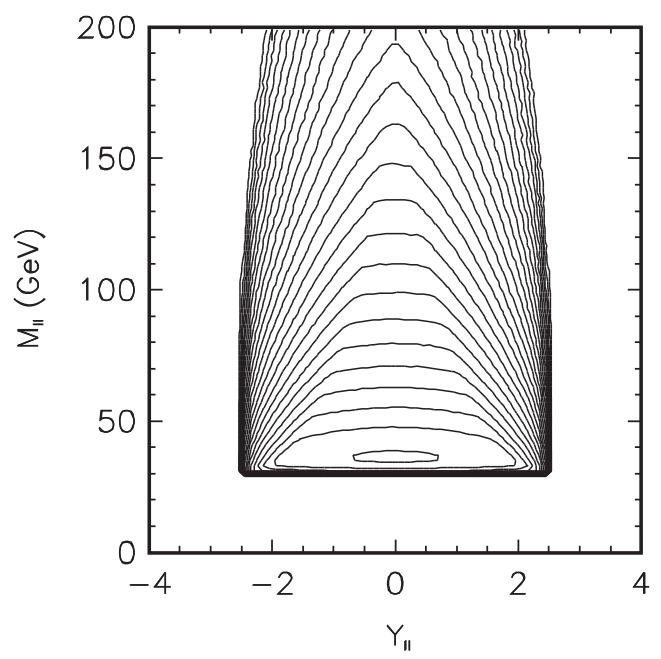

FIG. 4. Two-dimensional distribution in $\left(M_{l l}, Y_{l l}\right)$ for doubleelastic contribution. Here no cuts on neither $\xi_{1}$ nor $\xi_{2}$ were imposed. The $p_{t, \mu}>15 \mathrm{GeV}$ and $-2.5<y_{1}, y_{2}<2.5$ conditions were imposed here.

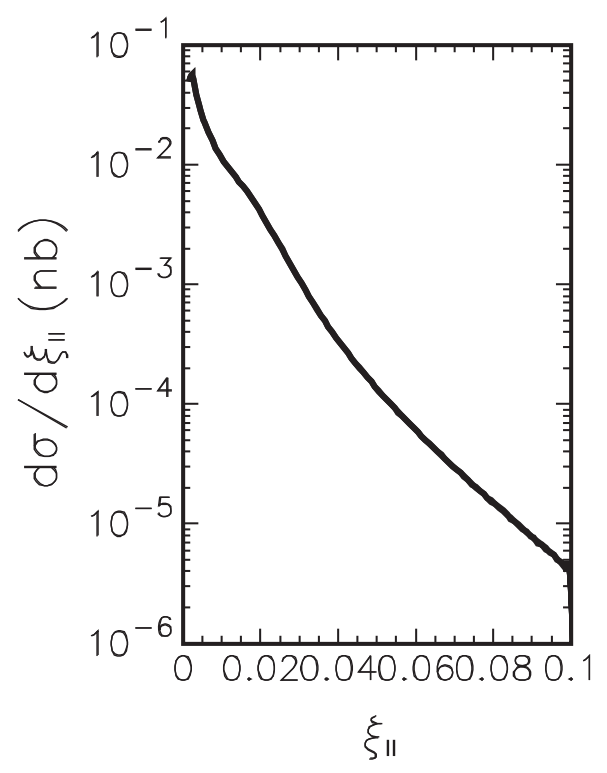

FIG. 5. $\xi_{1 / 2}$ distribution for dimuon production for the doubleelastic mechanism.

are very similar, at least for the relatively high cuts on electron/muon transverse momenta.

In Fig. 3 we present dimuon invariant mass distribution for the double-elastic mechanism. The lower limitation $M_{\min }=30 \mathrm{GeV}$ of the distribution is due to the $p_{t}>$ $15 \mathrm{GeV}$ cut.

Very interesting is the two-dimensional distribution in $\left(M_{l l}, Y_{l l}\right)[1]$. We show it for the double-elastic contribution in Fig. 4.

So far we did not include cuts on $\xi_{1}=\xi_{l l}^{+}$or $\xi_{2}=\xi_{l l}^{-}$. The distribution in $\xi_{l l}^{ \pm}$is shown in Fig. 5. It is a steeply falling function with increasing $\xi$.

A two-dimensional distribution $\left(\xi_{l l}^{+}, \xi_{l l}^{-}\right)$is shown in Fig. 6. A strong dependence on both $\xi_{l l}^{+}$and $\xi_{l l}^{-}$can be

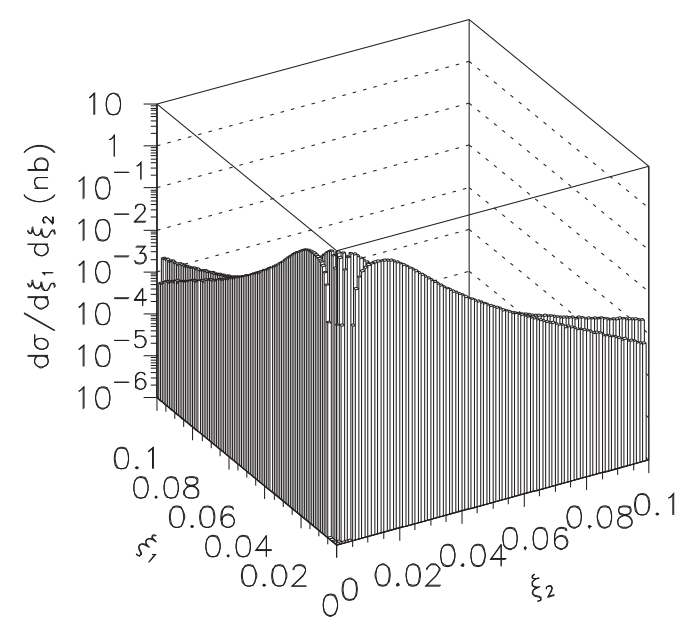

FIG. 6. Two-dimensional distribution in $\left(\xi_{l l}^{+}, \xi_{l l}^{-}\right)$for the doubleelastic mechanism. 


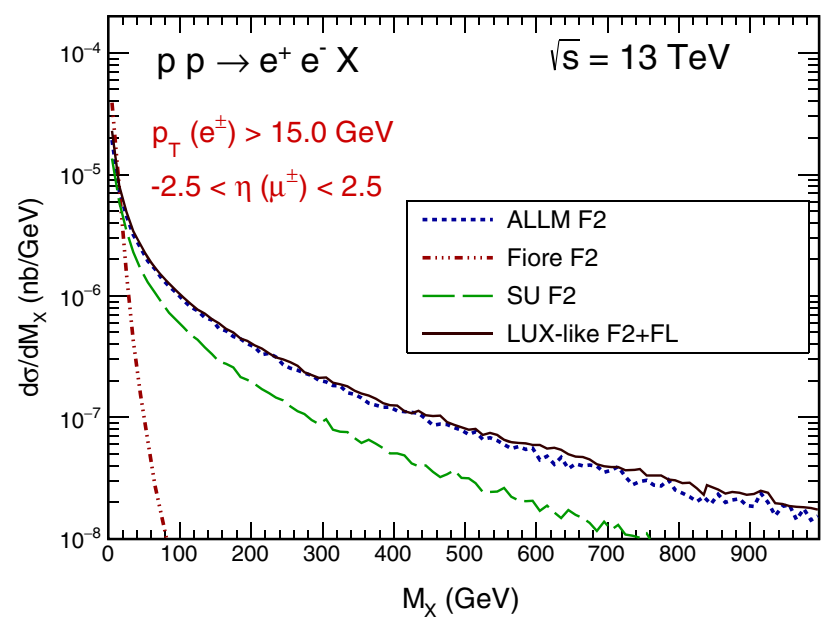

FIG. 7. Distribution in the mass of the baryonic remnant system $\left(M_{X}\right.$ or $\left.M_{Y}\right)$ for different structure functions from the literature. Here, in the case of the SU parametrization, only partonic contribution is included.

observed. This suggests that cuts on $\xi$ s will significantly lower the measured cross section.

\section{B. Single-dissociative contribution}

Now we wish to discuss similar distributions for singledissociative processes.

As will be discussed below the transverse momenta of initial (intermediate) photons in the inelastic vertex are large. We integrate over $q_{2 t} \in(0,100-500 \mathrm{GeV})$ for elastic-inelastic and $q_{1 t} \in(0,100-500 \mathrm{GeV})$ for inelasticelastic contribution.

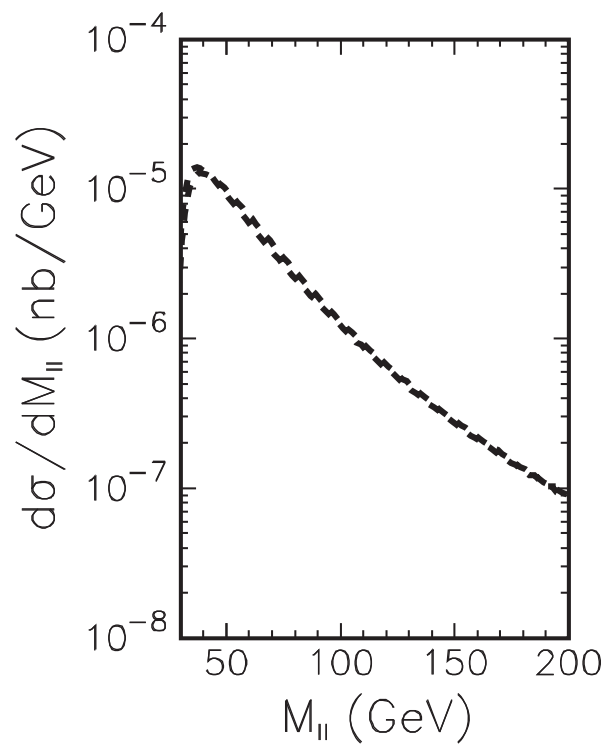

FIG. 8. Distribution in dilepton invariant mass for elasticinelastic and inelastic-elastic contributions. Here the SU structure function parametrization was used for example. Here the cuts on $\xi_{l l}^{+}$or $\xi_{l l}^{-}$are not imposed.

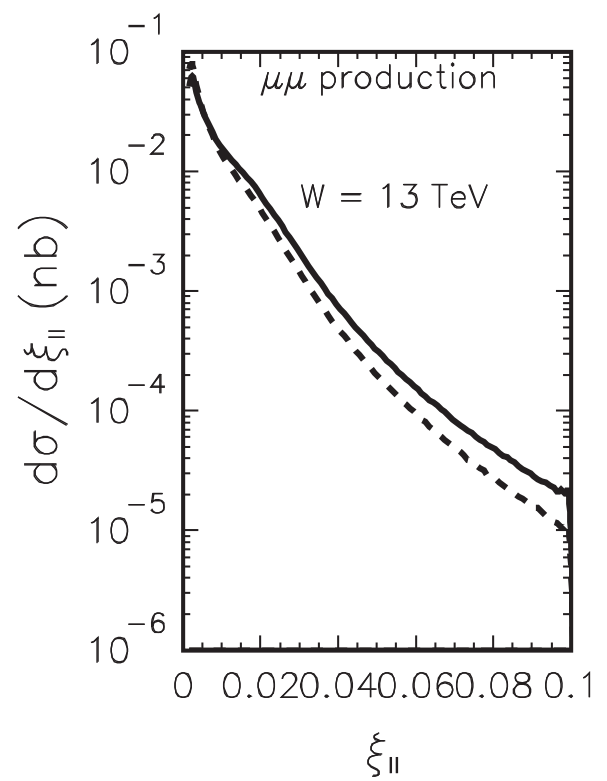

FIG. 9. Distribution in $\xi_{l l}^{ \pm}$for elastic-inelastic and inelasticelastic contributions. In this calculation the ALLM parametrization was used. The solid line is for right cuts on proton from the elastic vertex $\left(\xi_{l l}^{+}\right.$for elastic-inelastic and $\xi_{l l}^{-}$for inelastic-elastic contributions), the dashed line is for "incorrect" cuts on the inelastic system.

We start from the excitation of continuum. In the calculation below, for illustration we use the SzczurekUleshchenko deep-inelastic structure function [13] in calculating inelastic photon flux.

In Fig. 7 we show the distribution in the mass of the excited baryonic state. Here no extra cut on either $\xi_{i}$ or $p_{t, \text { pair }}\left(\vec{p}_{t \text {,pair }}=\vec{p}_{1}+\vec{p}_{2}\right)$ was imposed. The distributions for the ALLM and LUX-like structure functions are very

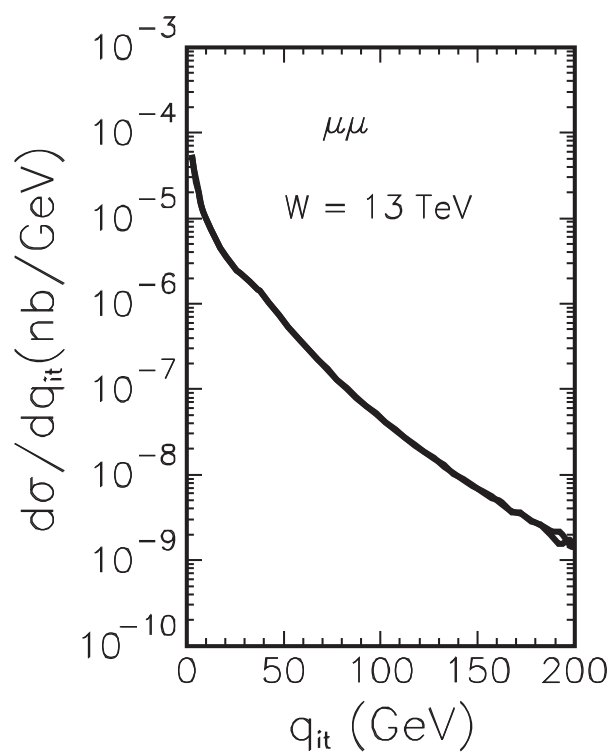

FIG. 10. Distribution in $q_{1 / 2, t}$ in the inelastic vertex. Here the ALLM parametrization is used. 

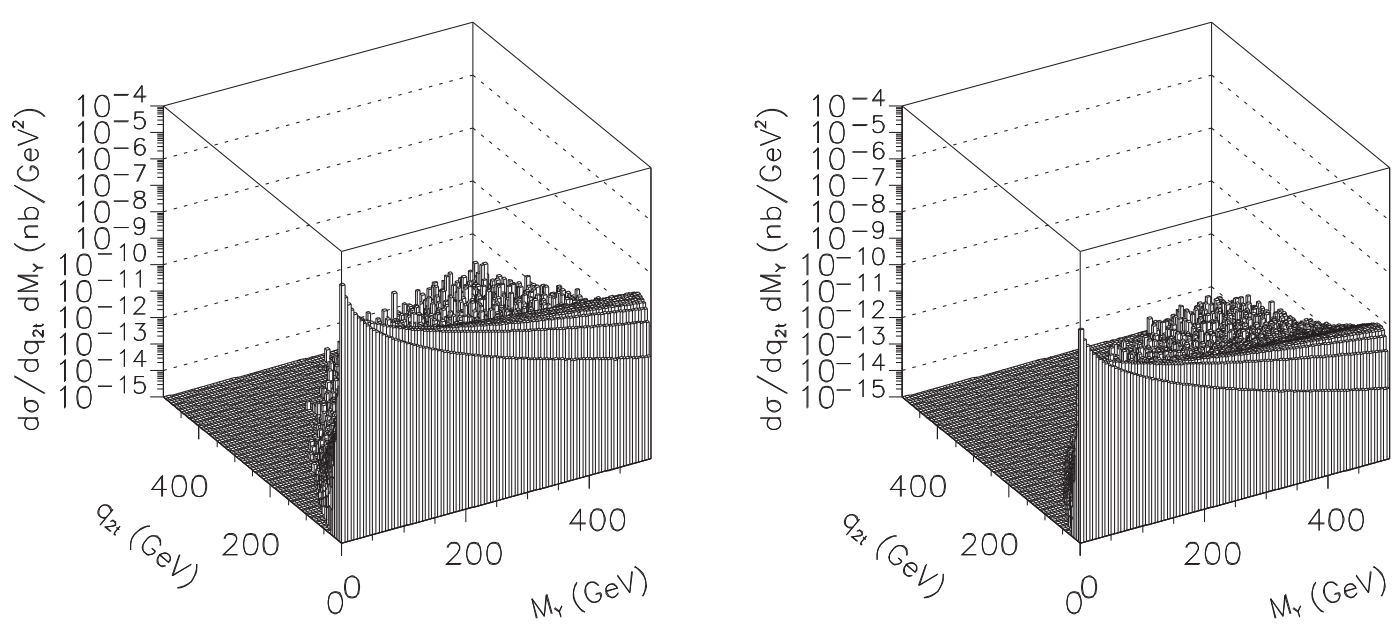

FIG. 11. Two-dimensional distribution in $\left(q_{2 t}, M_{Y}\right)$ for elastic-inelastic contribution. Similar distribution in $\left(q_{1 t}, M_{X}\right)$ plane can be obtained for the inelastic-elastic contribution. We show results without $\xi$ cut (left panel) and with $\xi$ cut (right panel).

similar and extend to very high $M_{X}$ (or $M_{Y}$ ) masses. The Fiore et al. structure function [14] does not lead to excitation of large $M_{X}$ and/or $M_{Y}$ and is therefore reliable only at low invariant masses. The reason is a missing in this parametrization [14] partonic contribution.
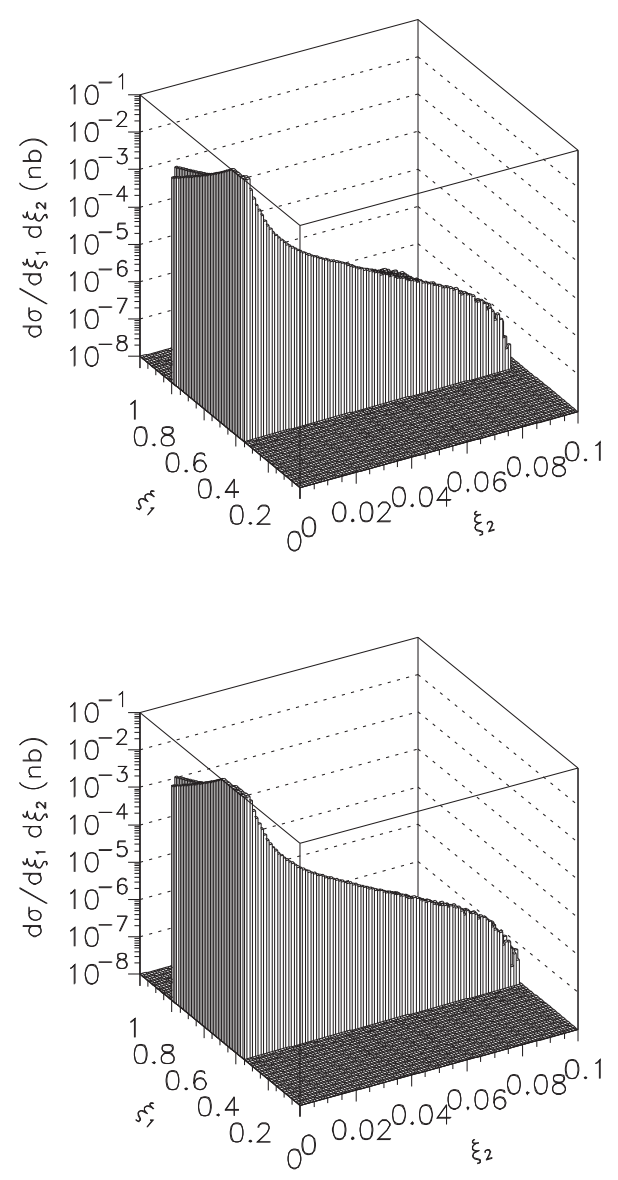

Now let us show some more differential distributions. In Fig. 8 we show dilepton invariant mass distribution without any cuts on $\xi_{l l}^{+}$or $\xi_{l l}^{-}$.

Finally in Fig. 9 we show distributions in $\xi_{l l}^{+}$or $\xi_{l l}^{-}$ for elastic-inelastic (black solid) and inelastic-elastic
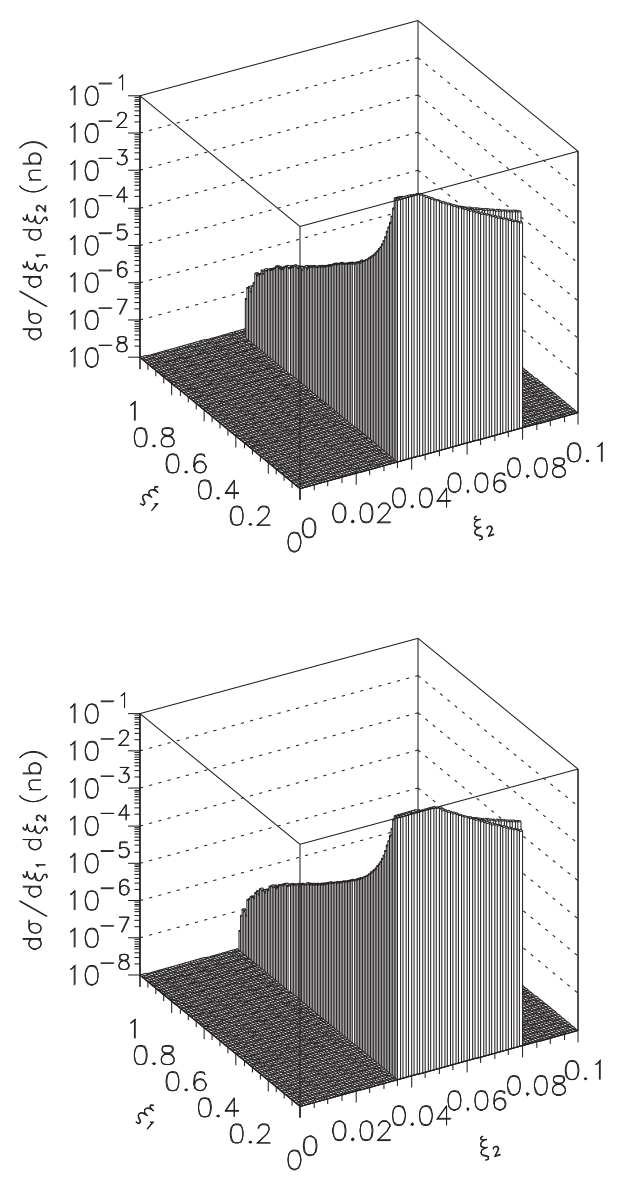

FIG. 12. Two-dimensional distributions in $\left(\xi_{l l}^{+}, \xi_{l l}^{-}\right)$for elastic-elastic, cut on 1 (upper-left corner), elastic-elastic, cut on 2 (upper-right corner), elastic-inelastic, cut on 1 (lower-left corner) and inelastic-elastic, cut on 2 (lower-right corner). Here the SU structure function was used for illustration. 
(black solid) contributions. For completeness we show also (dashed line) distributions in $\xi_{l l}$ corresponding to the remnant systems. The two distributions are fairly similar.

In Fig. 10 we show distribution in the initial photon transverse momentum in the inelastic vertex for elasticinelastic and inelastic-elastic contributions. The distributions extend to large transverse momenta.

Finally in this subsection we wish to discuss effect of correlations between $q_{2 t}$ and $M_{Y}$ for elastic-inelastic and $q_{1 t}$ and $M_{X}$ for inelastic-elastic contributions. In Fig. 11 we show only the first case. For small masses of the remnant only small $q_{2 t}$ are generated. In general, the larger $M_{Y}$ the larger $q_{2 t}$ can be generated. This shows that in the VEGAS integration one should carefully adjust the limits of integration on the $\left(q_{2 t}, M_{Y}\right)$ plane. There is no significant effect of the cut on the two-dimensional distribution.

\section{C. $\xi_{l l}^{+}$or $\xi_{l l}^{-}$cuts}

In the recent study presented in [1] only one proton was measured in AFP in order to get reasonable statistics. Typical experimental condition is: $\xi_{\min }<\xi_{i}<\xi_{\max }$. For the ATLAS apparatus it is [1] $\xi_{\min } \approx 0.035, \xi_{\max } \approx 0.08$. In the following we shall impose such a condition also in our calculations. Here we show how the $\xi_{l l}^{+}$and $\xi_{l l}^{-}$cuts work in practice.

In Fig. 12 we present two-dimensional distributions in $\xi_{l l}^{+}$and $\xi_{l l}^{-}$with appropriate cuts imposed to illustrate the large reduction of the cross section.

The ATLAS experimental cuts make the integrated cross section rather low compared to the result without such cuts as is shown in Table I.

The contribution of elastic-inelastic and inelastic-elastic mechanisms seem bigger than that of the elastic-elastic one. However, the situation changes when imposing extra cut on $p_{t, \text { pair }}$ (the numbers in paranthesis for single dissociative contributions). We present also naive, no $\Omega$ factor, see [13]. The $\Omega_{V}\left(x, Q^{2}\right)$ is a form factor modifying the naïve VDM (Vector Dominance Model) formula

$$
F_{2}^{N, \mathrm{VDM}}\left(x, Q^{2}\right)=\frac{Q^{2}}{\pi} \Sigma_{V} \frac{M_{V}^{4} \sigma_{V N}^{\text {tot }}}{\gamma_{V}^{2}\left(Q^{2}+M_{V}^{2}\right)^{2}} \Omega_{V}\left(x, Q^{2}\right),
$$

which depends on the time of life of the hadronic fluctuation. It modifies the VDM contribution at large Bjorken $x$. It was fitted to the structure function data in Ref. [13].

TABLE I. Integrated cross section for $\mu^{+} \mu^{-}$production in $\mathrm{fb}$ with one proton in the $0.035<\xi_{l l}^{ \pm}<0.08$ interval. In this calculation $p_{1 t}, p_{2 t}>15 \mathrm{GeV}$ and $-2.5<y_{1}, y_{2}<2.5$. No gap survival factor was imposed here. In the paranthesis we show result with extra $p_{t \text {,pair }}<5 \mathrm{GeV}$ condition. $2 \mathrm{UN}$ means the version of our code with doubly unintegrated photon distribution and GEN generator version of our code. In other cases singly unintegrated photon distribution is used from a simplified version of our code. The numbers in [...] were obtained with exact formula for $\xi_{1}$ and $\xi_{2}$.

\begin{tabular}{|c|c|c|}
\hline Contribution & c.s. in $\mathrm{fb}$ without $\xi$ cuts & c.s. in fb with $\xi$ cuts \\
\hline Elastic-elastic, cut on proton 1 & 358.68 & 5.4591 \\
\hline Elastic-elastic, cut on proton 2 & $\ldots . .$. & 5.4592 \\
\hline Elastic-inelastic, VDM (no $\Omega$ ), $0-100 \mathrm{GeV}$ & 98.0215 (2UN) & \\
\hline Inelastic-elastic, VDM (no $\Omega$ ), $0-100 \mathrm{GeV}$ & 98.0297 (2UN) & \\
\hline Elastic-inelastic SU partonic & $449.1076(2 \mathrm{UN})$ & \\
\hline Inelastic-elastic SU partonic & 449.0985 (2UN) & \\
\hline Elastic-inelastic, cut on proton 1, ALLM & $468.6102(2 \mathrm{UN})$ & 11.8292 \\
\hline Inelastic-elastic, cut on proton 2, ALLM & $468.6102(2 \mathrm{UN})$ & 11.8294 \\
\hline Elastic-inelastic, new Szczurek & $461.5330(2 \mathrm{UN})$ & $12.6046[14.1806](5.9311)$ \\
\hline Inelastic-elastic, new Szczurek & $461.5750(2 \mathrm{UN})$ & $12.6032[14.1806](5.9309)$ \\
\hline Elastic-inelastic, new Szczurek, $M_{Y}>500 \mathrm{GeV}$ & $\ldots$ & 0.7152 \\
\hline Inelastic-elastic, new Szczurek, $M_{X}>500 \mathrm{GeV}$ & $\ldots$ & 0.7149 \\
\hline Elastic-inelastic, ALLM & $571.871(\mathrm{GEN})$ & 9.711 \\
\hline Inelastic-elastic, ALLM & $571.562(\mathrm{GEN})$ & 9.621 \\
\hline Elastic-inelastic, LUX-like, $F_{2}+F_{L}$ & $635.215(\mathrm{GEN})$ & 19.894 \\
\hline Inelastic-elastic, LUX-like, $F_{2}+F_{L}$ & $635.102(\mathrm{GEN})$ & 19.831 \\
\hline Elastic-inelastic, LUX-like, $F_{2}$ only & ....... (GEN) & ...... \\
\hline Inelastic-elastic, LUX-like, $F_{2}$ only & $656.702(\mathrm{GEN})$ & $\ldots \ldots$ \\
\hline Elastic-inelastic, cut on proton 1 , resonances & 38.6709 (2UN) & 0.57872 \\
\hline Inelastic-elastic, cut on proton 2 resonances & $38.6639(2 \mathrm{UN})$ & 0.57872 \\
\hline Elastic-inelastic, cut on proton $1, \Delta^{+}$ & 28.5844 (2UN) & 0.42755 \\
\hline Inelastic-elastic, cut on proton $2 \Delta^{+}$ & $28.5814(2 \mathrm{UN})$ & 0.42763 \\
\hline
\end{tabular}




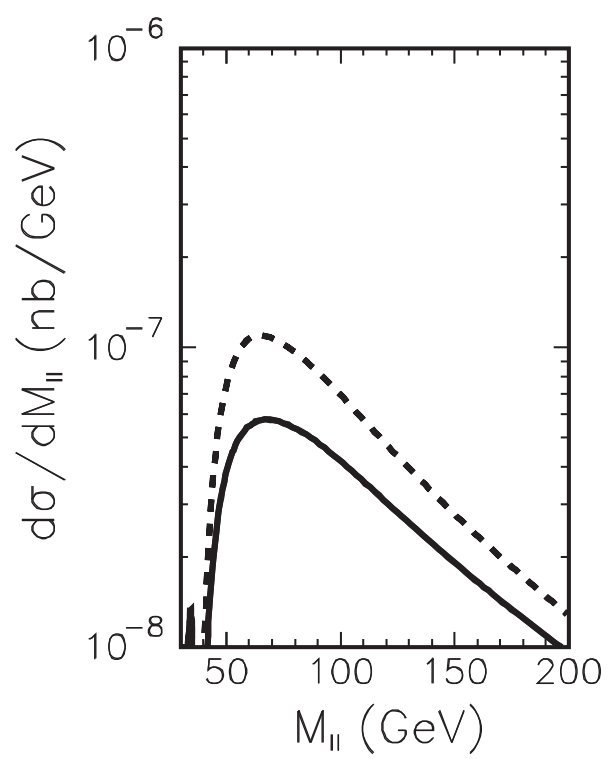

FIG. 13. Distribution in dilepton invariant mass for the different contributions considered. Here the cuts on $\xi_{l l}^{+}$or $\xi_{l l}^{-}$are imposed. The solid line is for double elastic contribution and the dashed line is for single dissociation contribution.

We have calculated also contribution of resonance excitations as parametrized in [14]. The resonance contribution $\left(\Delta^{+}\right.$etc.) is about $15 \%$ of the elastic-elastic contribution. The $\Delta^{+}$contribution is the dominant resonance contribution. We also use a popular in the context of HERA physics structure function parametrization of Abramowicz et al. [15]. We also show a result of a slightly modified SU parametrization. The new Szczurek parametrization includes partonic and VDM contributions as in the original Szczurek-Uleshchenko parametrization and includes in addition resonance contributions as parametrized by Fiore et al. [14]. The LUX-like structure function (see [16]) leads to largest cross section, especially when the cut on $\xi$ variable is imposed.

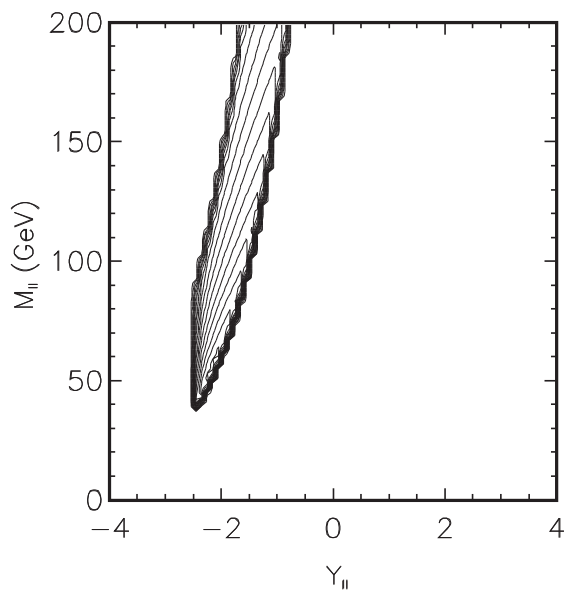

These results were obtained with the generator (GEN) version of our code. In the case of LUX-like model we included also longitudinal structure function $F_{L}$, which lowers the cross section (see the numbers in the table).

A technical remark is in order here. The range of integration in $q_{1 t}$ and $q_{2 t}$ is crucial to get correct result. The limits of integration should be different for elastic and inelastic arm. For double-elastic contribution it is sufficient to take $q_{i, t}<5 \mathrm{GeV}$ as an upper integration limit. For single dissociative contribution we integrate in the interval $q_{i, t}<100-500 \mathrm{GeV}$ or $q_{i, t}<100-500 \mathrm{GeV}$ for the inelastic arm. The first limit is enough when $p_{t, \text { pair }}<5 \mathrm{GeV}$ is imposed as in the ATLAS experiment, otherwise it should be a larger limit.

Now we wish to show other differential distributions for the case of including the experimental cuts on $\xi_{l l}^{ \pm}$. In Fig. 13 we show dilepton invariant mass distribution. The two coinciding solid lines correspond to elastic-elastic contributions, while the two dashed lines to single dissociative contributions.

The correlation in $\left(Y_{l l}, M_{l l}\right)$ becomes very interesting when cuts on $\xi$ are imposed.

Let us start from elastic-elastic contribution. We observe, see Fig. 14, two separate bands for conditions exclusively on $\xi_{1}$ (left panel) and for condition exclusively on $\xi_{2}$ (right panel). Only for extremely large $M_{l l}>200 \mathrm{GeV}$ one can satisfy both conditions simultaneously. However, there the cross section is exteremely small.

In Fig. 15 we show two-dimensional distribution in $\left(M_{l l}, Y_{l l}\right)$ for elastic-inelastic (left panel) and inelasticelastic (right panel) single dissociative production of the dimuon pairs. We impose condition on $\xi_{1}$ for elasticinelastic and on $\xi_{2}$ for inelastic-elastic contributions.

Let us look now at the projection on $Y_{l l}$. The distribution in rapidity of the pair $Y_{l l}$ is shown in Fig. 16. We observe clear symmetry with respect to $Y_{l l}=0$ for the two elastic-elastic contributions and similar symmetry between

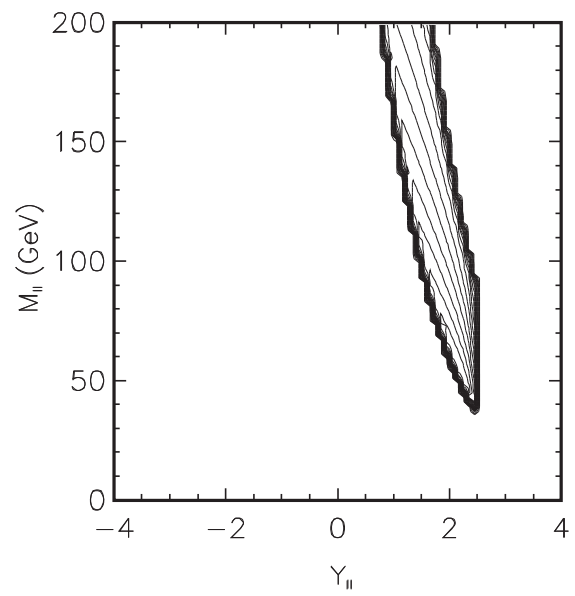

FIG. 14. Two-dimension distribution in $\left(M_{l l}, Y_{l l}\right)$ for double-elastic contribution. Here we have imposed experimental condition on $\xi_{2}$ (left panel) or $\xi_{1}$ (right panel) as explained in the main text. The $p_{t, \mu}>15 \mathrm{GeV}$ condition was imposed in addition. 

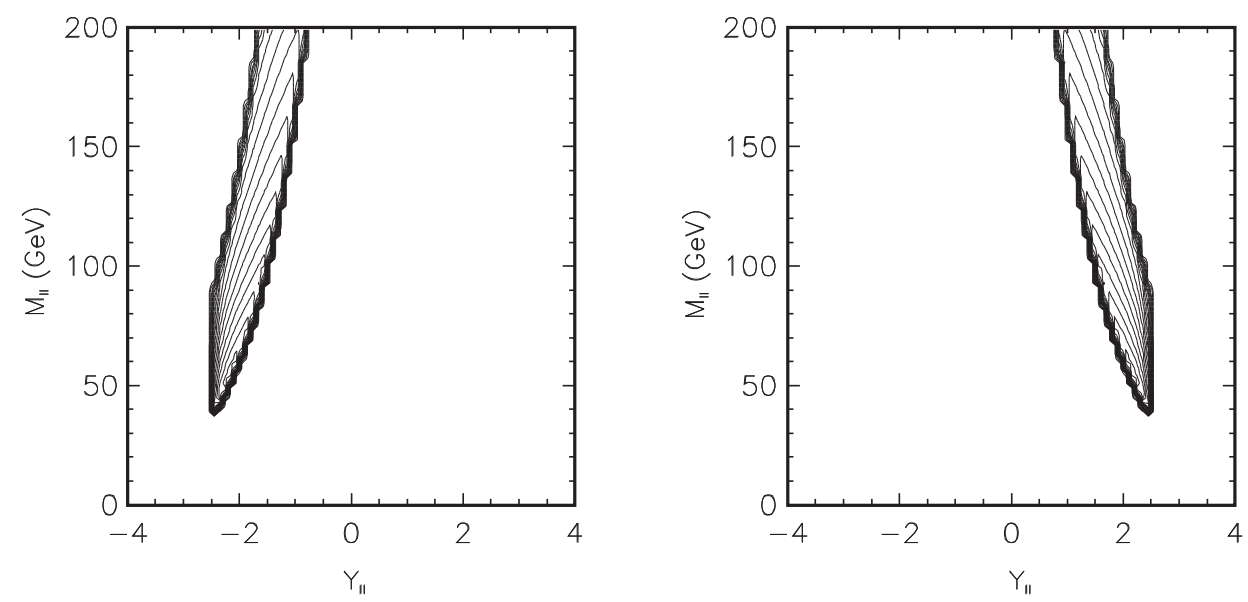

FIG. 15. Two-dimensional distribution in $\left(M_{l l}, Y_{l l}\right)$ for inelastic-elastic (left panel) and elastic-inelastic (right panel) contributions. The Szczurek-Uleshchenko structure function parametrization was used here for illustration. Here we have imposed experimental condition on $\xi_{2}$ (left panel) or $\xi_{1}$ (right panel) as explained in the main text. The $p_{t, \mu}>15 \mathrm{GeV}$ condition has been imposed in addition.

elastic-inelastic and inelastic-elastic contributions. The contribution related to the cut on $\xi_{l l}^{+}$or $\xi_{l l}^{-}$are almost totally separated. This is a reason of a dip observed in $d \sigma / d Y_{l l}$ at $Y_{l l}=0$ by the ATLAS collaboration [1].

A final fully leptonic variable we wish to consider in the context of the $\xi$ cut is $p_{t, \text { diff }}$, where $\vec{p}_{t \text {,diff }}=\vec{p}_{1 t}-\vec{p}_{2 t}$. We show corresponding distributions in Fig. 17. The biggest effect of the cut is for small values of $p_{t, \text { diff }}$.

What are typical $x_{B j}$ and $Q^{2}$, i.e., arguments of the structure functions for the considered processes with single dissociation (inelastic-elastic or elastic-inelastic processes) is shown in Fig. 18. Both perturbative $\left(Q^{2}>2 \mathrm{GeV}^{2}\right)$ and nonperturbative $\left(Q^{2}<2 \mathrm{GeV}^{2}\right)$ regions enter the

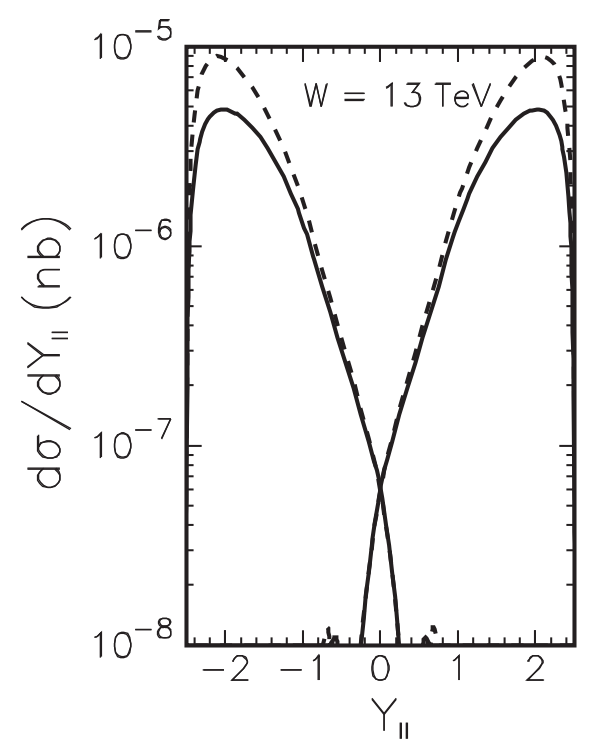

FIG. 16. Distribution in dilepton rapidity for four different contributions considered. Here the cuts on $\xi_{l l}^{+}$or $\xi_{l l}^{-}$are imposed. The solid line is for double elastic contribution and the dashed line is for single dissociation contribution. corresponding calculations. The nonperturbative region is even relatively larger when the cut on $p_{t \text {,pair }}<5 \mathrm{GeV}$ is imposed as in the recent ATLAS [1] paper.

In Fig. 19 we show distribution (projection of the previous two-dimensional distribution) in $x_{B j 1}$ or $x_{B j 2}$ for the ALLM parametrization without (solid line) and with (dashed line) cuts on $\xi$. The two distributions have rather similar shape which means that a similar range of $x_{B j 1}$ or $x_{B j 2}$ is tested in both cases. Here the most probable range of $x_{B j 1}$ or $x_{B j 2}$ is about $10^{-2}$, the region of experiments on deep-inelastic scattering performed in the past by the NMC collaboration. In the $\gamma \gamma \rightarrow l^{+} l^{-}$process one is not sensitive to a small $x_{B j}$ region $\left(x_{B j}<10^{-3}\right)$. For comparison we also show distribution for the resonant contributions

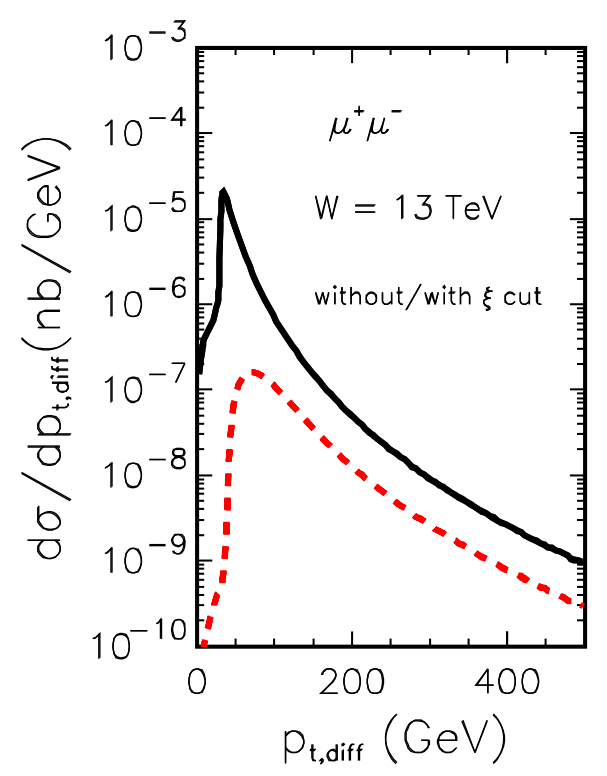

FIG. 17. Distribution in $p_{t, \text { diff }}$ without (upper solid coinciding curves) and with (lower dashed coinciding curves) $\xi$ cuts. 

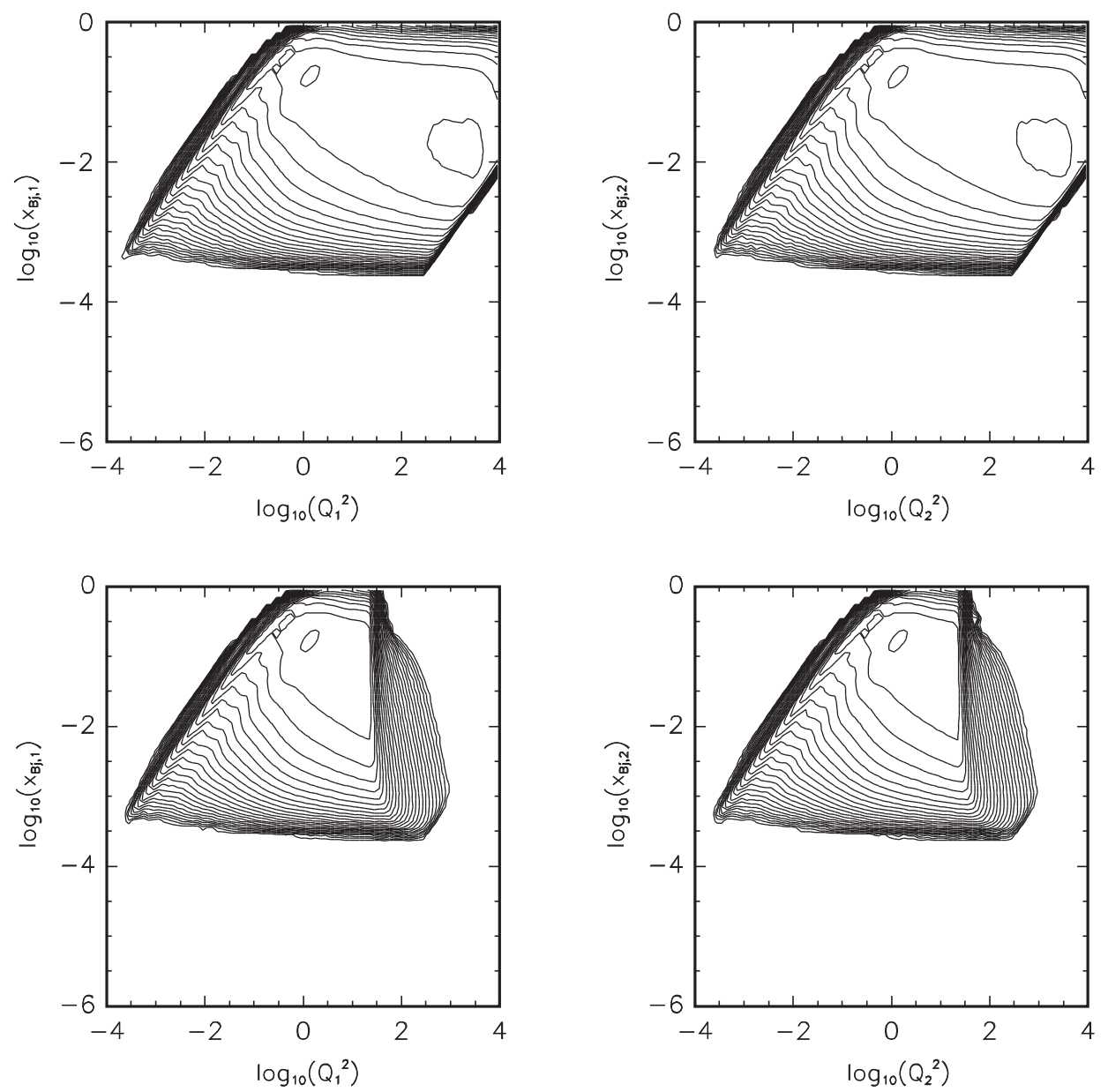

FIG. 18. The range in the $\left(x_{B j}, Q^{2}\right)$ space tested in inelastic-elastic (left) and elastic-inelastic DIS processes with $\xi$ cuts. The lower panels include also an extra cut $p_{t, \text { pair }}<5 \mathrm{GeV}$.

that are not explicit in most of the parametrizations used in the literature.

In Fig. 20 we show complementary distribution in the second argument of the structure function $\left(Q_{1}^{2}\right.$ or $\left.Q_{2}^{2}\right)$. Both small (nonperturbative) and large (perturbative) $Q_{i}^{2}$ occur. The nonperturbative region is relatively larger when the experimental cut on $p_{t \text {,pair }}<5 \mathrm{GeV}$ [1] is imposed.

How the acoplanarity distribution depends on cuts is illustrated in Fig. 21. We show result without any cut, with $\xi$ cut and with additional condition on the pair transverse momentum. The condition on the pair transverse momentum significantly change the distribution. Also photon final state radiation may be important in this context but this goes beyond the scope of the present analysis.

The single dissociative process leads to an emission of a (mini)jet (see Fig. 22) which produces hadrons (mostly pions) that may destroy the rapidity gap if required experimentally. Here we wish to show rapidity distributions of such jets separately for elastic-inelastic and inelasticelastic contributions. In Fig. 23 we show results without and with cut on $\xi_{1 / 2}$.
The emitted jet when entering the main detector (ATLAS or CMS) will destroy rapidity gap and such cases are usually vetoed in experiment. Here we wish to show what are correlations of jet rapidity with mass of the remnant. In Fig. 24 we show such correlations for inelastic-elastic (left panel) and elastic-inelastic (right panel) contributions. If the mass of the remnant system is small the corresponding jet is emitted outside of the main detector and is therefore not observed. On the contrary the jets corresponding to large masses enter the main detector and destroy the rapidity gap.

\section{SUPERCHIC results and gap survival factor}

For comparison we did also calculations using a popular SUPERCHIC program [11]. In this code the soft gap survival factor can be included. In this code the absorption effects can be included and the corresponding soft gap survival factor, or even a survival function, defined as

$$
S_{G}\left(\zeta_{1}, \ldots, \zeta_{n}\right)=\frac{\left.\frac{d \sigma}{d \zeta_{1} \ldots d \zeta_{n}}\right|_{\text {withSR }}}{\left.\frac{d \sigma}{d \zeta_{1} \ldots d \zeta_{n}}\right|_{\text {withoutSR }}}
$$




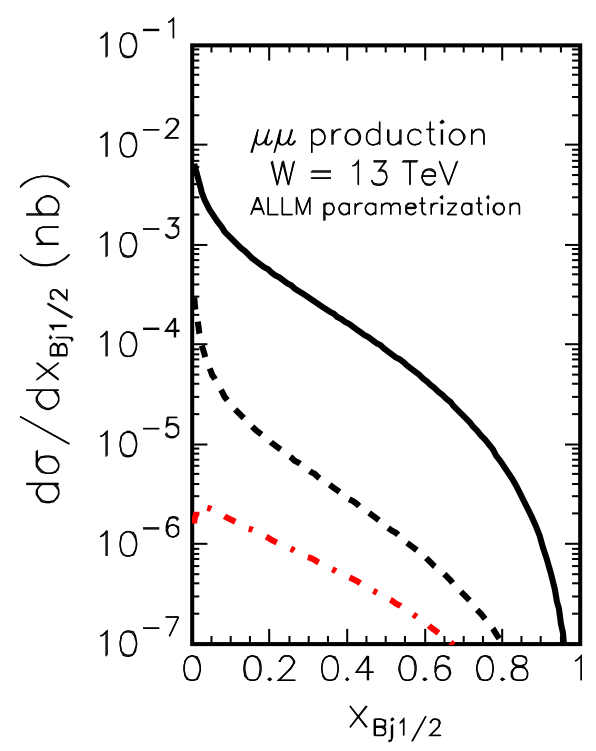

FIG. 19. Distribution in $x_{B j}$ for single dissociative process. Shown are results without (solid line) and with (dashed line) cuts on longitudinal momentum fraction $\xi$. In this calculation the ALLM (see Ref. [15]) parametrization of $F_{2}$ structure function is used. For completeness we show also the contribution of proton resonances with cuts on $\xi$ (red dash-dotted line).

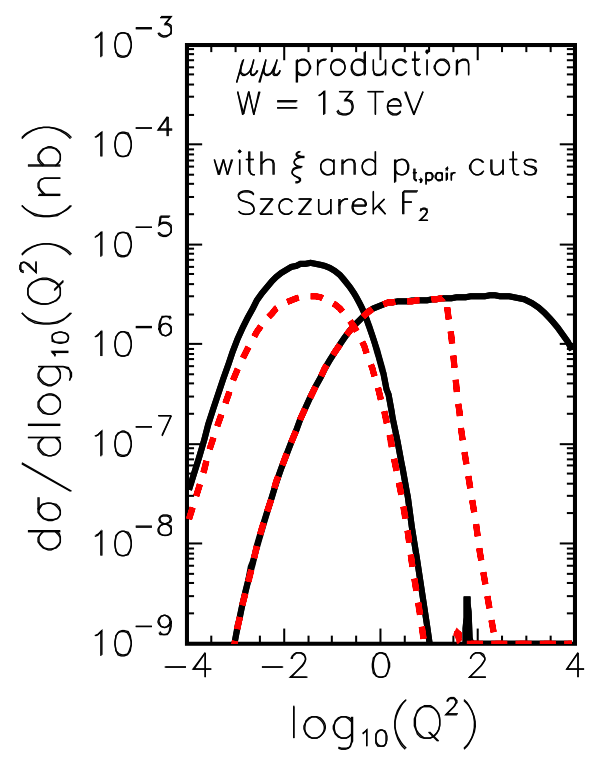

FIG. 20. Distribution in $\log _{10}\left(Q_{i}^{2}\right)$ for single dissociative process with the cut on $\xi$. We show distributions for elastic (left) and inelastic (right) vertex. In this calculation the new Szczurek parametrization of $F_{2}$ was used. We also show similar distributions with the upper cut on $p_{t \text {,pair }}$ (red dashed line) as applied in the recent ATLAS measurement [1].

can be calculated as a function of $\zeta_{1}, \ldots, \zeta_{n}$, which are arbitrary kinematical variables. Some examples will be presented below.

We wish to study how the effective gap survival factor changes with implementation of the cut on $\xi_{1}$ or $\xi_{2}$. In the

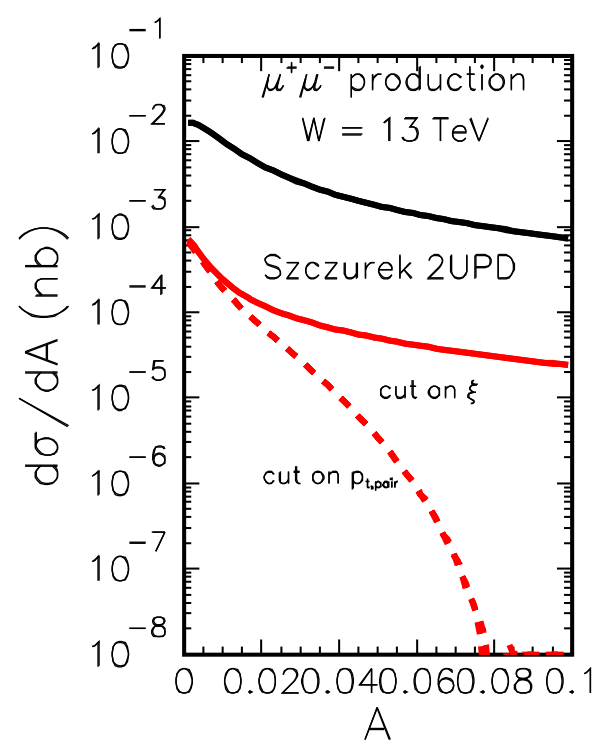

FIG. 21. Acoplanarity distribution for single dissociative contributions without any (upper black solid curve), with $\xi$ cut (middle red solid curve) and with extra $p_{t \text {,pair }}<5 \mathrm{GeV}$ condition (lower red dashed curve). In this calculation the new Szczurek parametrization of $F_{2}$ (including resonances) was used.

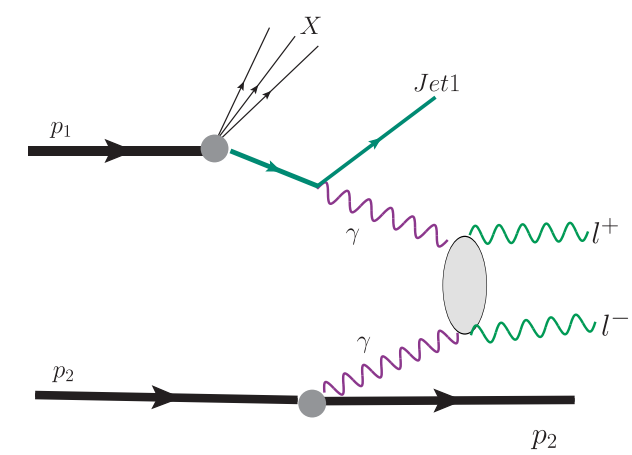

FIG. 22. Diagram with (min)jet production due to quark/ antiquark knockout.

SUPERCHIC generator the gap survival is calculated with opacity obtained within two-channel Good-Walker formalism. The parameters of the model were obtained in [17]. The results presented here were obtained with the event sample $N_{\text {event }}=50000$, unless otherwise stated.

The integrated cross sections are collected in Table II. The cross sections obtained in SUPERCHIC are somewhat larger than those obtained using our code (compare the numbers in Table II with the numbers in Table I). This is partially due to different structure functions used in both codes. The cuts on $\xi$ in this table were calculated as

$$
\xi_{1 / 2}=\frac{\sqrt{s} / 2-E_{3 / 4}}{\sqrt{s} / 2}
$$

where $E_{3}$ or $E_{4}$ are energies of outgoing protons. 


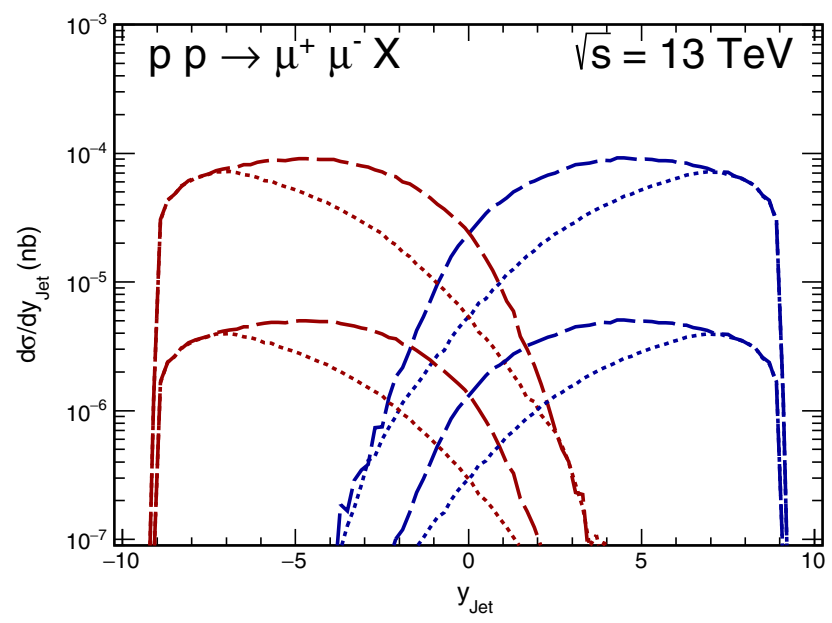

FIG. 23. Distribution in rapidity of (mini)jets for inclusive case (upper curves) and for the case with cut on $\xi_{1 / 2}$ (lower curves). The extra dotted lines represent results that include the cut $p_{t, \text { pair }}<5 \mathrm{GeV}$ as in the ATLAS analysis [1].

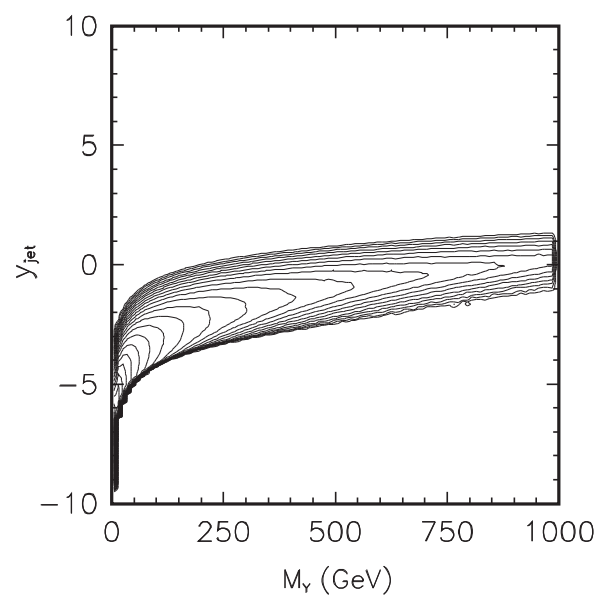

FIG. 24. Remnant mass-jet rapidity correlations. The standard $\xi$ cuts were applied here.

The typical cuts on $\xi_{1}$ or $\xi_{2}$ lower the cross section by almost two orders of magnitude. The numbers obtained here (SUPERCHIC) after the $\xi$ cuts included are, however, significantly larger than their counterparts in Table I. The soft gap survival factor strongly depends on whether we have fully elastic or single dissociation process. This is related to typical transverse momenta of outgoing protons that are bigger for processes with proton dissociation. Similar tendencies can be observed when cuts on fractional longitudinal momentum loss of protons is imposed. Then, however, the soft gap survival factors are significantly reduced $(0.931 \rightarrow 0.886$ for double elastic contribution and $0.738 \rightarrow 0.521$ for processes with single dissociation). The effect of explicit cuts on $y_{1}$ and $y_{2}$ is small and leads to slight increase compared to the case of an internal cut on $Y_{l l}$ imposed by default in SUPERCHIC.
TABLE II. Integrated cross section for $\mu^{+} \mu^{-}$production in $\mathrm{pb}$ for $\sqrt{s}=13 \mathrm{TeV}$ using the SUPERCHIC program [11]. We show results without any extra external cuts (upper part), with extra cut on individual rapidities $-2.5<y_{1}, y_{2}<2.5$ (middle part) and with extra cut on $\xi$ (lower part). The cut on $\xi_{i}$ is $0.035<\xi_{l l}^{ \pm}<0.08$. In addition, in this calculation $p_{1 t}, p_{2 t}>15 \mathrm{GeV}$. We show result with (first column) and without (second column) soft gap survival. To calculate absorption effects we used model no 4 as implemented in the SUPERCHIC generator (see also [17]). In the last column we show average gap survival factor being the ratio of the cross sections in previous two columns. In all cases $p_{1 t}, p_{2 t}>15 \mathrm{GeV}$. The numbers with (*) in the last block were obtained with 10000 events only.

\begin{tabular}{lccc}
\hline \hline Reaction & No soft $S_{G}$ & With soft $S_{G}$ & $\left\langle S_{G}\right\rangle$ \\
\hline$-2.5<Y_{l l}<2.5$ & & & \\
Elastic-elastic & 0.54438 & 0.50402 & 0.926 \\
Inelastic-elastic & 0.89595 & 0.64283 & 0.717 \\
Elastic-inelastic & 0.89587 & 0.64254 & 0.717 \\
Inelastic-inelastic & 1.62859 & 0.24172 & 0.148 \\
$-2.5<y_{1}, y_{2}<2.5$ & & & \\
$\quad$ in addition & & & \\
Elastic-elastic & 0.42268 & 0.39355 & 0.931 \\
Inelastic-elastic & 0.69241 & 0.51092 & 0.738 \\
Elastic-inelastic & 0.69246 & 0.51087 & 0.738 \\
$\xi$ cut in addition & & & \\
Elastic-elastic, cut on $\xi_{1}$ & 0.00762 & 0.00675 & 0.886 \\
Elastic-elastic, cut on $\xi_{2}$ & 0.00762 & 0.00675 & 0.886 \\
Inelastic-elastic, cut on $\xi_{2}$ & 0.02496 & 0.01324 & 0.530 \\
Elastic-inelastic, cut on $\xi_{1}$ & 0.02393 & 0.01238 & 0.517 \\
$p_{t, \text { pair }<5 \text { GeV in addition }}$ & & & \\
Elastic-elastic & $\ldots \ldots$. & $\ldots \ldots$ & \\
Inelastic-elastic, cut on $\xi_{2}$ & 0.00807 & $0.00437(*)$ & 0.541 \\
Elastic-inelastic, cut on $\xi_{1}$ & 0.00807 & $0.00437(*)$ & 0.542 \\
\hline \hline
\end{tabular}

Now we shall discuss the effect of gap survival factor on differential distributions. In this case we shall use event sample generated by the SUPERCHIC 4 generator. For this purpose we generated five $10^{+4}$ events and wrote a simple code that prepares distributions of interest. The statistics is rather small so the differential distributions will fluctuate much more in comparison to distributions obtained from our codes based on the VEGAS algorithm.

In Fig. 25 we present distribution in dimuon invariant mass for the case without $\xi$ cuts (left panel) and with $\xi$ cuts (right panel). The elastic-elastic (dashed line) and elasticinelatic + inelastic-elastic (solid line) are shown separately. On average we observe larger invariant masses in the case with $\xi$ cuts.

In Fig. 26 we present similar distributions but in $p_{t \text {,pair }}$. The shapes of distributions obtained with and without soft gap survival effects seems rather similar.

In Fig. 27 we present similar distributions but in $Y_{l l}$. Without the $\xi$ cut we observe quite different shapes of 

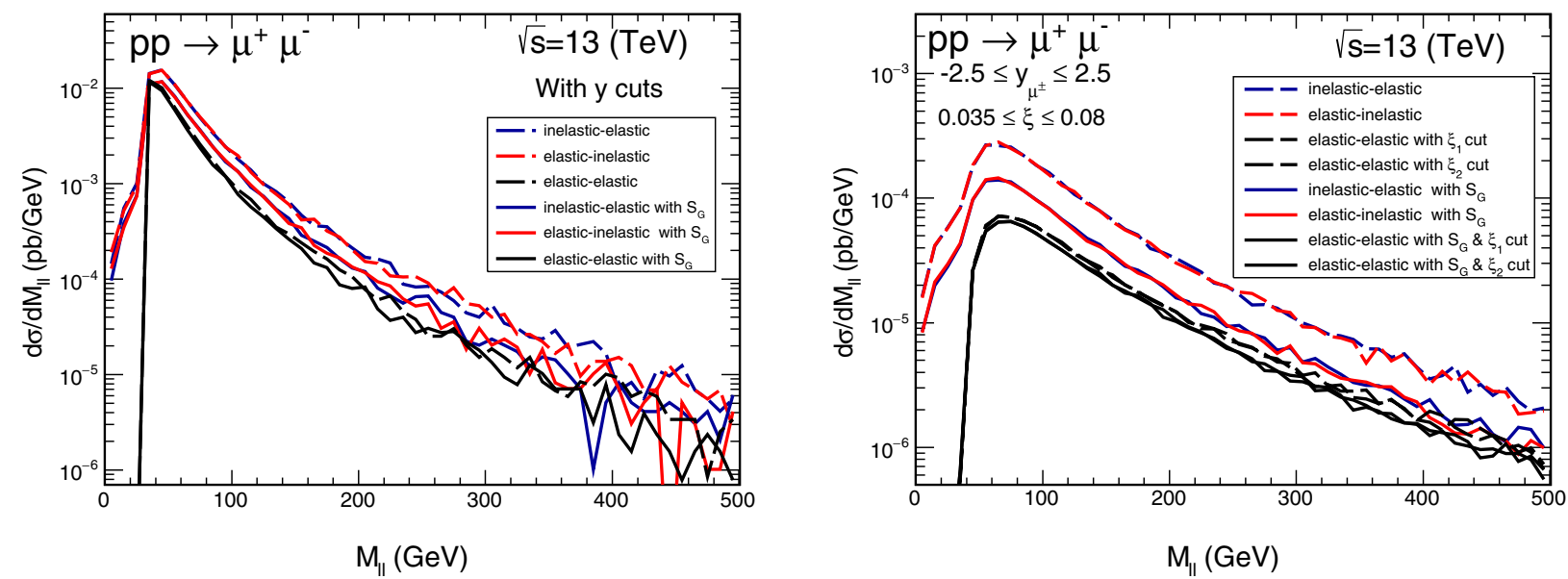

FIG. 25. Distribution in dimuon invariant mass for the different contributions considered. We consider the case without $\xi$ cuts (left panel) and with $\xi$ cuts (right panel).
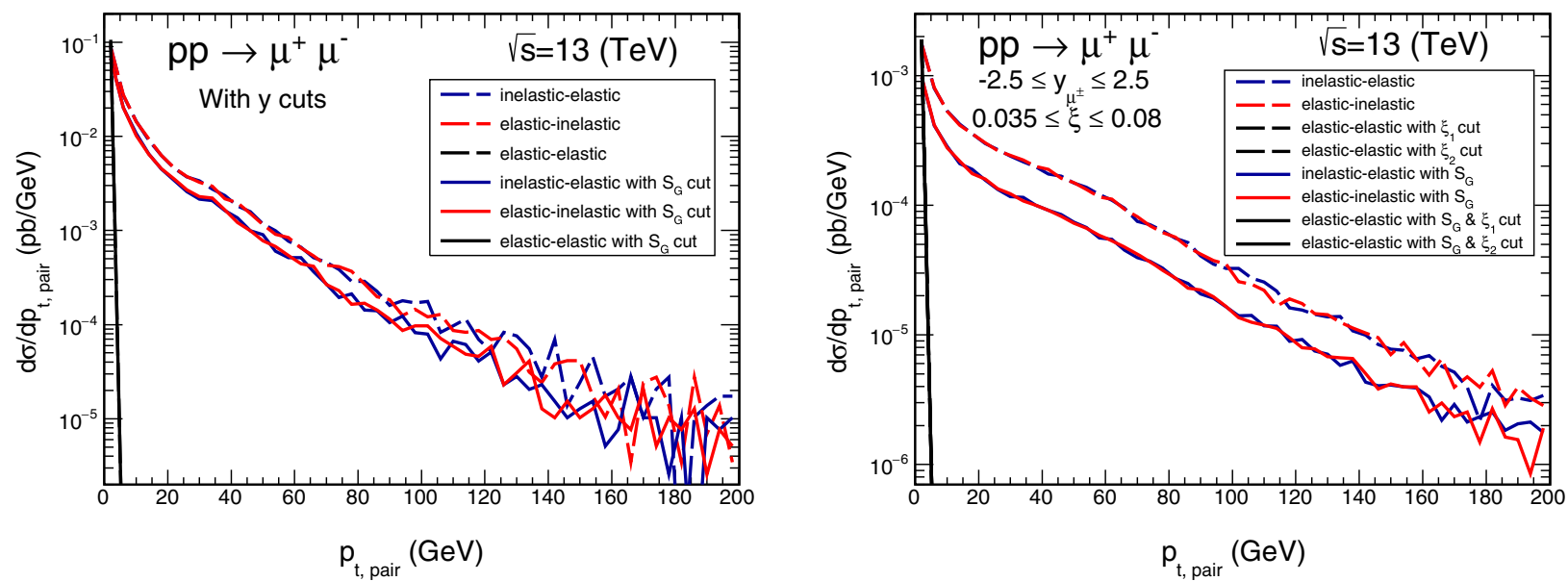

FIG. 26. Distribution in dimuon transverse momentum for the different contributions considered. We consider the case without $\xi$ cuts (left panel) and with $\xi$ cuts (right panel).
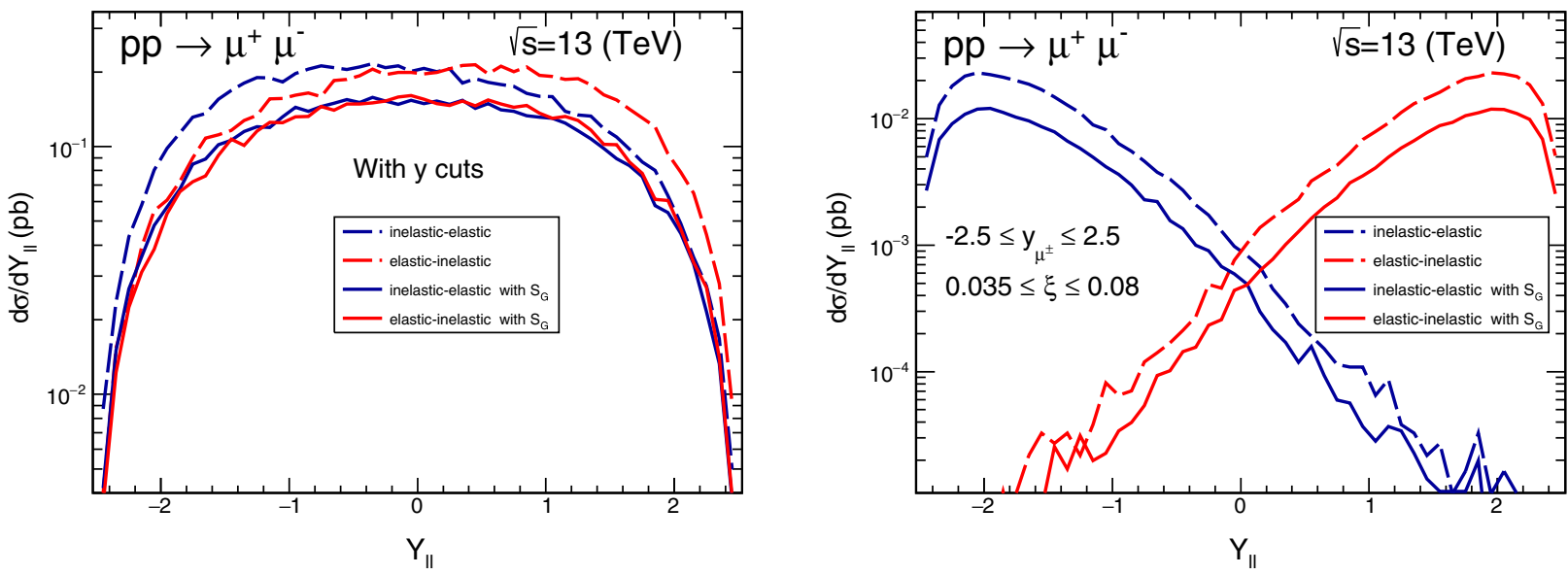

FIG. 27. Distribution in rapidity of the dimuon pair. We show the case without $\xi$ cuts (left panel) and with $\xi$ cuts (right panel) for the different contributions considered. 
distributions in $Y_{l l}$ without and with soft rapidity gap survival factor (see the left panel). When the $\xi$ cut is imposed the distributions with and without soft rapidity gap survival factor have very similar shapes. Then, however, the elastic-inelastic and inelastic-elastic contributions are well separated in $Y_{l l}$. The sum of both contributions has a characteristic dip at $Y_{l l}=0$. This is the same as was discussed in the previous section.

In Fig. 28 we show corresponding gap survival factor calculated as

$$
\begin{aligned}
S_{G}\left(M_{l l}\right) & =\frac{d \sigma /\left.d M_{l l}\right|_{\text {withSR }}}{d \sigma /\left.d M_{l l}\right|_{\text {withoutSR }}}, \\
S_{G}\left(p_{t, \text { pair }}\right) & =\frac{d \sigma /\left.d p_{t, \text { pair }}\right|_{\text {withSR }}}{d \sigma /\left.d p_{t, \text { pair }}\right|_{\text {withoutSR }}},
\end{aligned}
$$

the ratio of the cross section with the soft rapidity gap survival factor to its counterpart without including the effect, differential in $M_{l l}$ (left panel) or in $p_{t \text {,pair }}$ (right panel) for double elastic (dashed line) and single dissociation (solid line). We observe a small dependence on both $M_{l l}$ and on $p_{t, \text { pair }}$. The gap survival factor for double elastic component is larger than that for single dissociation. The gap survival factor corresponding to the measurement of one proton is significantly smaller than that for the inclusive case. The rather large fluctuations are due to limited statistics (50 000 events).

In Fig. 29 we show in addition soft gap survival factor as a function of the rapidity of the dimuon pair. We observe a strong dependence of the gap survival factor on $Y_{l l}$ separately for elastic-inelastic and inelastic-elastic components but only in the case when proton is not measured. This effect may be very difficult to address experimentally as in this (no proton measurement) case one measures the sum of the both (all) components, where the effect averages and becomes more or less independent of $Y_{l l}$ (see black dash-dotted curve). However, it seems interesting to understand the dependence on $Y_{l l}$ for individual component from theoretical point of view.
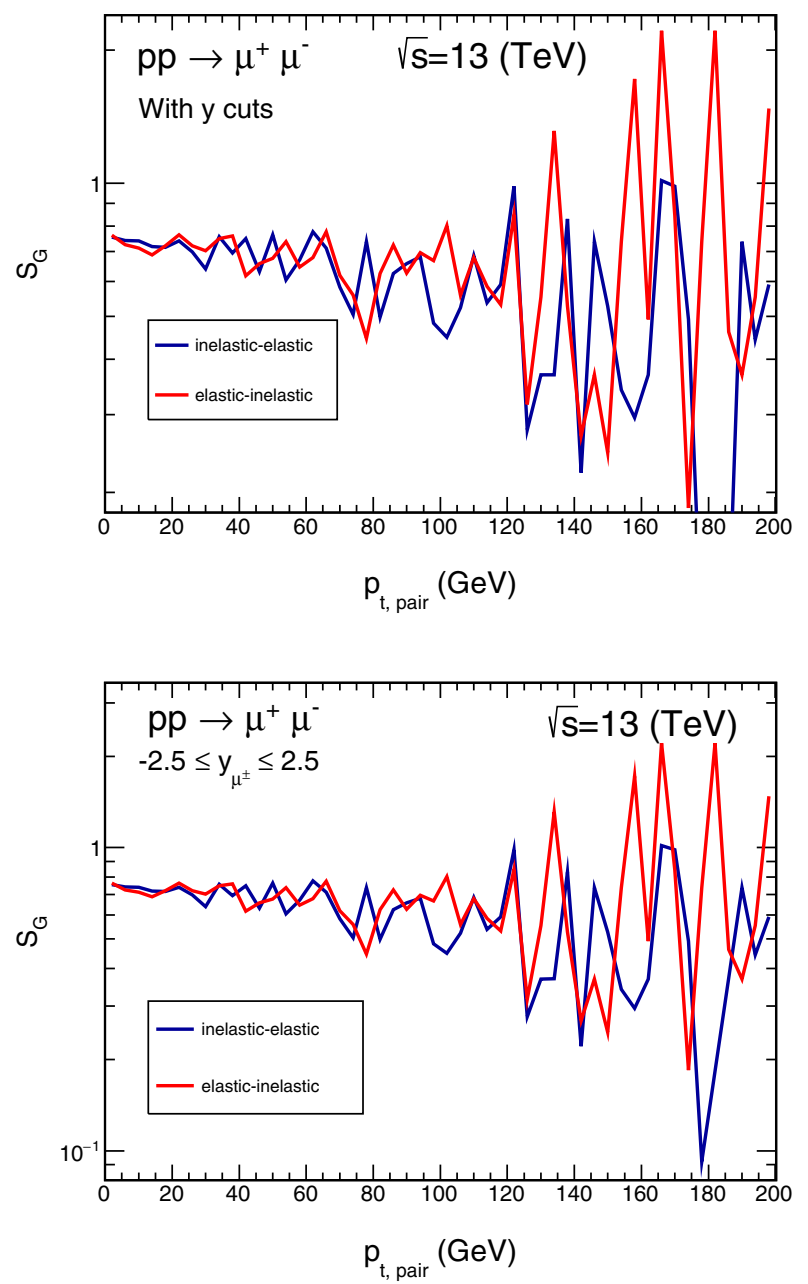

FIG. 28. The soft gap survival factor as a function of dilepton invariant mass (left panels) and as a function of transverse momentum of the pair (right panels) for single dissociation (solid line) mechanisms. We show the result without $\xi$ cuts (upper panels) and with $\xi$ cuts (lower panels). 

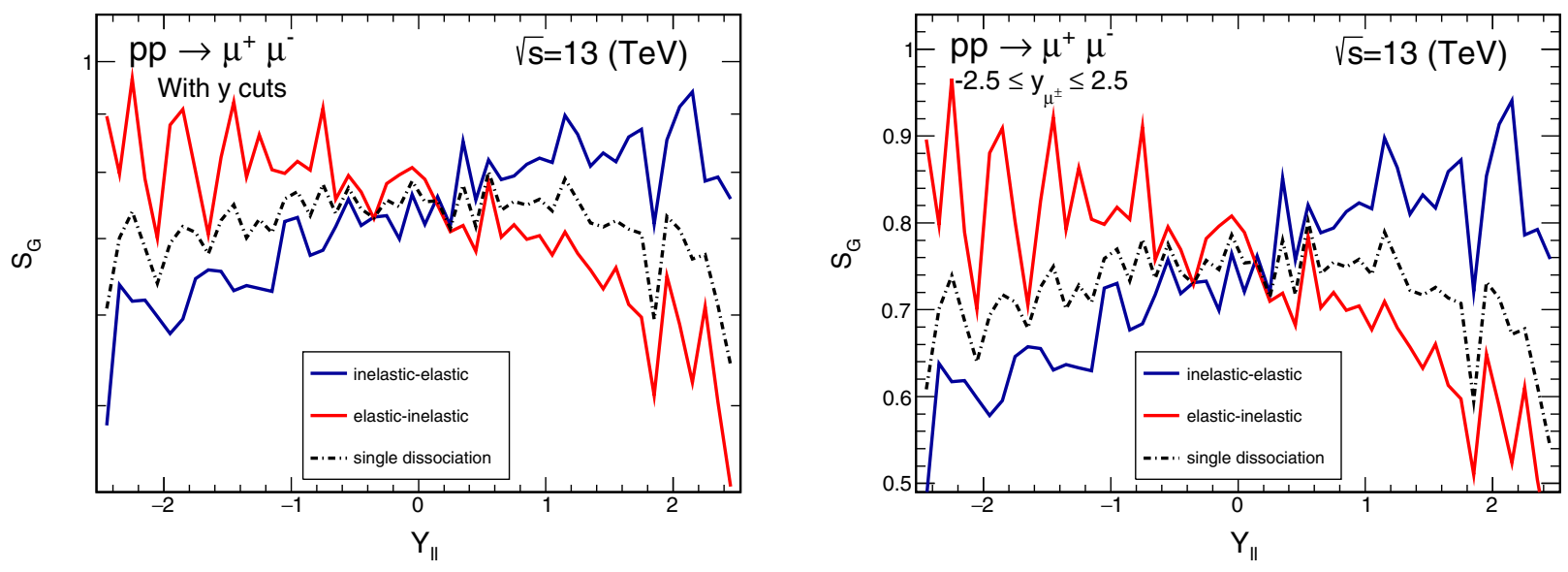

FIG. 29. The soft gap survival factor as a function of rapidity of the $\mu^{+} \mu^{-}$pair for single proton dissociation. We show the result without $\xi$ cuts (left panel) and with $\xi$ cuts (right panel). The dash-dotted black line represents effective gap survival factor for both singledissociation components added together.
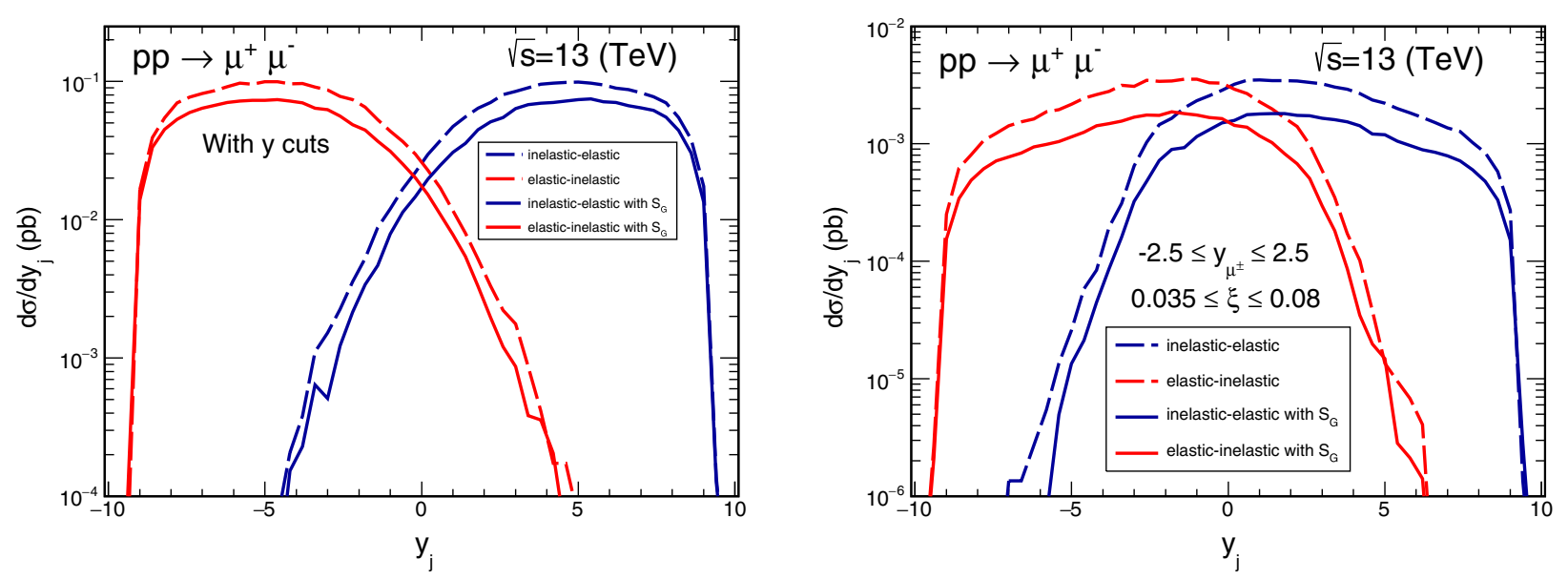

FIG. 30. Distribution in the (mini)jet rapidity for the inclusive case with no $\xi$ cut (left panel) and when the cut on $\xi$ is imposed (right panel) for elastic-inelastic and inelastic-elastic contributions as obtained from the SUPERCHIC generator. We show result without (dashed line) and with (solid line) soft rescattering correction.

Finally we wish to discuss how the proton dissociation further reduces the gap survival factor due to emission of a (mini)jet that can enter into the main detector and destroy the rapidity gap. ${ }^{1}$ This was discussed, e.g., in $[6,7,18]$. In Fig. 30 we show the (mini)jet distribution in rapidity for elastic-inelastic and inelastic-elastic components. We show the distribution without imposing the $\xi$ cut (left panel) and when imposing the $\xi$ cut (right panel). One can observe slightly different shape for both cases. The corresponding gap survival factor (probability of no jet in the main detector) is 0.8 and 0.5 , respectively. The probability of no emission around the $\gamma \gamma \rightarrow \mu^{+} \mu^{-}$vertex is, however,

\footnotetext{
${ }^{1}$ However, the rapidity gap condition was not explicitly imposed in recent analyses with forward proton measurements in either [2] or [1].
}

much more difficult to calculate and requires inclusion of remnant hadronization that is model dependent.

In Table III we show probability that the (mini)jet is outside the main detector: i.e., $y_{\text {jet }}<-2.5$ or $y_{\text {jet }}>2.5$. The numbers below are similar in size to the soft gap survival factor collected in Table II. Imposing cuts on $\xi$ lowers the corresponding (minijet) rapidity gap survival factor while imposing an extra cut $p_{t \text {,pair }}<5 \mathrm{GeV}$, as in the ATLAS experiment, increases it back.

The factor below must be evidently included in the case when rapidity gap condition is imposed experimentally. It is less clear what to do when the condition of separated $\mu^{+}$ and $\mu^{-}$are imposed as in the ATLAS experiment [1]. In the following we assume that the particles from (mini)jet, emitted from the same vertex as leptons, will always break the conditions, provided they are emitted in the same range of rapidities as the measured leptons. This range is defined 
TABLE III. Gap survival factor due to minijet emission. The first block is with only internal SUPERCHIC cut: $-2.5<Y_{l l}<2.5$, the second block is when the condition on individual rapidities is imposed extra, the third block includes in addition the cut on $\xi_{1}$ or $\xi_{2}$, and the final block includes also the condition $p_{t, \text { pair }}<5 \mathrm{GeV}$. In all cases $p_{1 t}, p_{2 t}>15 \mathrm{GeV}$. In the last panel (*) means 10000 events only.

\begin{tabular}{lcc}
\hline \hline Contribution & Without $S_{G}$ & With $S_{G}$ \\
\hline Cut on $Y_{l l}$ only & & \\
Elastic-inelastic & 0.76304 & 0.78756 \\
Inelastic-elastic & 0.76278 & 0.78898 \\
Cut on $y_{1}$ and $y_{2}$ in addition & & \\
Elastic-inelastic & 0.77366 & 0.79250 \\
Inelastic-elastic & 0.76926 & 0.78744 \\
Cut on $\xi_{1}$ or $\xi_{2}$ in addition & & \\
Elastic-inelastic & & \\
Inelastic-elastic & 0.52430 & 0.53976 \\
Cut on $p_{t, \text { pair in addition }}$ & 0.53118 & 0.53614 \\
Elastic-inelastic & & \\
Inelastic-elastic & 0.83144 & $0.84350\left(^{*}\right)$ \\
\hline \hline
\end{tabular}

by the geometry of the main ATLAS (CMS) detector. In real experiment [1] one imposes rather condition on $R\left(\right.$ track, $\left.l^{+}\right)>R_{0}$ and $R\left(\right.$ track, $\left.l^{-}\right)>R_{0}$ (no emission in the cones around both leptons). In the ATLAS experiment $R_{0}=0.01$ was used. It would be interesting to study experimentally the gap survival factor as a function of $R_{0}$.

\section{CONCLUSIONS}

In the present paper we have discussed dilepton production via photon-photon fusion with one forward proton, which can be measured in forward detectors such as the AFP for the ATLAS experiment. We have considered both double-elastic and single-dissociative contributions (it was argued that the contribution of double dissociation is negligible when forward proton is measured). In the latter case we have considered both continuum production as well as $\Delta^{+}$isobar production or production of other nucleon resonances. The continuum contribution is calculated for different parametrizations of the deep-inelastic structure functions from the literature. The differences of the cross section are of the order of $10-20 \%$ and can be regarded as uncertainties of the present modelling.

We have imposed conditions on $\xi_{1}$ or $\xi_{2}$ for the forward emitted protons. Several distributions have been shown and discussed in this case. Particularly interesting is the distribution in $Y_{l l}$ which has a minimum at $Y_{l l} \sim 0$. The minimum at $Y_{l l}=0$ is caused by the experimental condition on $\xi_{l l}^{ \pm}$imposed on the leading proton.

We have also quantified the region of $x_{B j}$ (the argument of the structure functions) relevant for the $p p \rightarrow l^{+} l^{-}$ processes. We found that the typical values of $x_{B j}$ are rather larger than $10^{-3}$ both for inclusive case and in the case with proton measurement.

We have also made calculations with the popular SUPERCHIC generator and compared corresponding results to the results of our code(s). In general, the results are very similar.

We have also calculated the soft rapidity gap survival factor [probability of no hadron emission in the range of the main (ATLAS, CMS) detector] as a function of $M_{l l}$, the transverse momentum of the dilepton pair, and the mass of the proton remnant and $Y_{l l}$. No evident dependencies on the variables have been found for the single dissociation, except of distribution in $Y_{l l}$. We have found different (much larger) gap survival factor for fully elastic contribution than for single proton dissociation. The soft gap survival factor for single dissociative contribution strongly depends on whether proton is measured or not. It is significantly smaller when the forward proton is measured.

We have performed analysis of the range of arguments of the structure functions relevant for photon-photon processes. The photon virtualities are both in perturbative and nonperturbative regions. The nonperturbative region becomes relatively larger when the cut $p_{t \text {,pair }}<5 \mathrm{GeV}$, as imposed in the recent ATLAS analysis [1].

We have also calculated gap survival factor due to mini(jet) emission by checking whether the minijet enters or not the main detector. The second type of the gap survival also strongly depends on whether the outgoing proton is measured or not. It is about 0.8 for inclusive case (no proton measurement) and about 0.5 for the case with proton measurement in the forward proton detector (with typical limited $\xi$ values). The second type of gap survival factor is to large extent independent of the soft gap survival factor, so in general the two factors can be included multiplicatively. In our opinion it is not clear, however, what to do in the case when lepton isolation cuts with specific parameters are included. Then inclusion of both effects multiplicatively may lead to underestimation of the measured cross section.

In the present paper we have intentionally concentrated on discussing effects related to a measurement of one forward proton, i.e., on imposing $\xi$ cuts and absorption effect and not on direct comparison to the new ATLAS data.

\section{ACKNOWLEDGMENTS}

This study was partially supported by the Polish National Science Center Grant No. UMO-2018/31/B/ST2/03537 and by the Center for Innovation and Transfer of Natural Sciences and Engineering Knowledge in Rzeszów. We are indebted to Jesse Liu, Rafał Staszewski, and Marek Tasevsky for a discussion and explanation of details of the recent ATLAS paper. 
[1] G. Aad et al. (ATLAS Collaboration), Observation and Measurement of Forward Proton Scattering in Association with Lepton Pairs Produced via the Photon Fusion Mechanisms at ATLAS, Phys. Rev. Lett. 125, 261801 (2020).

[2] A. M. Sirunyan et al. (CMS Collaboration), Observation of proton-tagged, central (semi)exclusive production of highmass lepton pairs in $\mathrm{p} \mathrm{p}$ collisions at $13 \mathrm{TeV}$ with the CMSTOTEM precision proton spectrometer, J. High Energy Phys. 07 (2018) 153.

[3] G. G. da Silveira, L. Forthomme, K. Piotrzkowski, W. Schäfer, and A. Szczurek, Central $\mu^{+} \mu^{-}$production via photon-photon fusion in proton-proton collisions with proton dissociation, J. High Energy Phys. 02 (2015) 159.

[4] M. Łuszczak, W. Schäfer, and A. Szczurek, Two-photon dilepton production in proton-proton collisions: Two alternative approaches, Phys. Rev. D 93 (2016) 074018.

[5] M. Łuszczak, W. Schäfer, and A. Szczurek, Production of $W^{+} W^{-}$pairs via $\gamma^{*} \gamma^{*} \rightarrow W^{+} W^{-}$subprocess with photon transverse momenta, J. High Energy Phys. 05 (2018) 064.

[6] L. Forthomme, M. Łuszczak, W. Schäfer, and A. Szczurek, Rapidity gap survival factors caused by remnant fragmentation for $W^{+} W^{-}$pair production via $\gamma^{*} \gamma^{*} \rightarrow W^{+} W^{-}$subprocess with photon transverse momenta, Phys. Lett. B 789, 300 (2019).

[7] M. Luszczak, L. Forthomme, W. Schäfer, and A. Szczurek, Production of $t \bar{t}$ pairs via $\gamma \gamma$ fusion with photon transverse momenta and proton dissociation, J. High Energy Phys. 02 (2019) 100.

[8] P. Lebiedowicz and A. Szczurek, Exclusive and semiexclusive production of $\mu^{+} \mu^{-}$pairs with Delta isobars and other resonances in the final state and the size of absorption effects, Phys. Rev. D 98, 053007 (2018).
[9] P. Lebiedowicz and A. Szczurek, Exclusive production of heavy charged Higgs boson pairs in the $p p \rightarrow p p H^{+} H^{-}$ reaction at the LHC and a future circular collider, Phys. Rev. D 91, 095008 (2015).

[10] M. Dyndal and L. Schoeffel, The role of finite-size effects on the spectrum of equivalent photons in proton-proton collisions at the LHC, Phys. Lett. B 741, 66 (2015).

[11] L. A. Harland-Lang, M. Tasevsky, V. A. Khoze, and M. G. Ryskin, A new approach to modelling elastic and inelastic photon-initiated production at the LHC: SuperChic 4, Eur. Phys. C 80, 925 (2020).

[12] T. Ahmed et al. (H1 Collaboration), Deep inelastic scattering events with a large rapidity gap at HERA, Nucl. Phys. B429, 477 (1994).

[13] A. Szczurek and V. Uleshchenko, Nonpartonic components in the nucleon structure functions at small $Q * * 2$ in the broad range of x, Eur. Phys. J. C 12, 663 (2000).

[14] R. Fiore, A. Flachi, L. L. Jenkovszky, A. I. Lengyel, and V. K. Magas, Explicit model realizing parton hadron duality, Eur. Phys. J. A 15, 505 (2002).

[15] H. Abramowicz and A. Levy, The ALLM parameterization of sigma(tot)(gamma* p): An update, arXiv:hep-ph/ 9712415.

[16] A. V. Manohar, P. Nason, G. P. Salam, and G. Zanderighi, The photon content of the proton, J. High Energy Phys. 12 (2017) 046.

[17] V. A. Khoze, A. D. Martin, and M. G. Ryskin, Diffration at the LHC, Eur. Phys. J. C 73, 2503 (2013).

[18] L. A. Harland-Lang, V. A. Khoze, and M. G. Ryskin, The photon PDF in events with rapidity gap, Eur. Phys. J. C 76, 255 (2016). 